\title{
Prevention of connexin-43 remodeling protects against Duchenne muscular dystrophy cardiomyopathy
}

\author{
Eric Himelman, ${ }^{1}$ Mauricio A. Lillo, ${ }^{2}$ Julie Nouet, ${ }^{1}$ J. Patrick Gonzalez, ${ }^{1}$ Qingshi Zhao, ${ }^{1}$ Lai-Hua Xie, ${ }^{1}$ Hong Li, ${ }^{3}$ Tong Liu, ${ }^{3}$ \\ Xander H.T. Wehrens, ${ }^{4}$ Paul D. Lampe, ${ }^{5}$ Glenn I. Fishman, ${ }^{6}$ Natalia Shirokova, ${ }^{2}$ Jorge E. Contreras, ${ }^{2}$ and Diego Fraidenraich ${ }^{1}$ \\ 'Department of Cell Biology and Molecular Medicine, ²Department of Pharmacology, Physiology and Neuroscience, and ${ }^{3}$ Center for Advanced Proteomics Research, New Jersey Medical School, Rutgers \\ Biomedical and Health Sciences, Newark, New Jersey, USA. ${ }^{4}$ Department of Molecular Physiology and Biophysics, Medicine, Neuroscience, and Pediatrics, Cardiovascular Research Institute, Baylor College of \\ Medicine, Houston, Texas, USA. ${ }^{5}$ Fred Hutchinson Cancer Research Center, Translational Research Program, Public Health Sciences Division, Seattle, Washington, USA. 'Leon H. Charney Division of Cardiology, \\ New York University Langone Health, New York, New York, USA
}

\begin{abstract}
Aberrant expression of the cardiac gap junction protein connexin-43 (Cx43) has been suggested as playing a role in the development of cardiac disease in the mdx mouse model of Duchenne muscular dystrophy (DMD); however, a mechanistic understanding of this association is lacking. Here, we identified a reduction of phosphorylation of Cx43 serines S325/ S328/S330 in human and mouse DMD hearts. We hypothesized that hypophosphorylation of Cx43 serine-triplet triggers pathological Cx43 redistribution to the lateral sides of cardiomyocytes (remodeling). Therefore, we generated knockin mdx mice in which the Cx43 serine-triplet was replaced with either phospho-mimicking glutamic acids (mdxS3E) or nonphosphorylatable alanines (mdxS3A). The mdxS3E, but not mdxS3A, mice were resistant to Cx43 remodeling, with a corresponding reduction of $\mathrm{Cx} 43$ hemichannel activity. MdxS3E cardiomyocytes displayed improved intracellular $\mathrm{Ca}^{2+}$ signaling and a reduction of NADPH oxidase 2 (NOX2)/ROS production. Furthermore, mdxS3E mice were protected against inducible arrhythmias, related lethality, and the development of cardiomyopathy. Inhibition of microtubule polymerization by colchicine reduced both NOX2/ROS and oxidized CaMKII, increased S325/S328/S330 phosphorylation, and prevented Cx43 remodeling in mdx hearts. Together, these results demonstrate a mechanism of dystrophic $\mathrm{Cx} 43$ remodeling and suggest that targeting $\mathrm{C} \times 43$ may be a therapeutic strategy for preventing heart dysfunction and arrhythmias in DMD patients.
\end{abstract}

\section{Introduction}

Duchenne muscular dystrophy (DMD) is an X-linked neuromuscular disease characterized by muscle susceptibility to mechanically induced damage, resulting in deterioration and degeneration (1). It affects 1 in every 3,500-5,000 male births and is considered the most common and fatal form of muscular dystrophy (2). DMD is caused by the absence of the protein dystrophin, a critical component of the dystrophin-glycoprotein complex (DGC) in skeletal and cardiac muscle $(3,4)$. Strategies such as adeno-associated virus (AAV) gene therapy, to deliver shortened yet functional versions of dystrophin (5), and CRISPR/Cas9 gene editing, to skip or restore mutated dystrophin gene sequences (6), possess great clinical potential to combat DMD. Nonetheless, concerns remain regarding the ability to produce body-wide effects with gene therapy and the potential for off-target effects of CRISPR/Cas9, emphasizing the continued need to focus on secondary molecular targets in

\section{Related Commentary: p. 1608}

Conflict of interest: The authors have declared that no conflict of interest exists. Copyright: (c) 2020, American Society for Clinical Investigation. Submitted: February 15, 2019; Accepted: December 18, 2019; Published: February 24, 2020.

Reference information: J Clin Invest. 2020;130(4):1713-1727. https://doi.org/10.1172/JCl128190.
DMD. Improvements in therapeutic treatments that combat skeletal muscle and respiratory dysfunctions have significantly prolonged DMD patients' lives (7). As a result, dystrophic cardiomyopathy is now the leading cause of death (8). However, the role of dystrophin in cardiac muscle differs from that in skeletal muscle and pathological mechanisms of DMD cardiomyopathy remain largely undefined, leaving a large unmet clinical need (9).

Connexins are emerging as therapeutic targets for heart failure (10). In the heart, connexins are responsible for maintaining electrical conduction by facilitating the rapid propagation of action potentials, leading to synchronous contraction (11). Six connexin proteins assemble to form one hemichannel, while two hemichannels from neighboring cardiomyocytes join to form a complete gap junction channel at the intercalated disc (ID). Connexin-43 (Cx43) is the most abundant connexin protein in the ventricular myocardium (12). In the heart, $\mathrm{Cx} 43$ is a short-lived protein that undergoes numerous posttranslational modifications (PTMs), including phosphorylation of the intracellular carboxyl terminus $(13,14)$. PTMs regulate many aspects of the Cx43 life cycle, notably the redistribution of $\mathrm{Cx} 43$ away from IDs and toward the lateral cardiomyocyte sarcolemma in a phenomenon termed gap junctional remodeling $(14,15)$. At these lateral sites, Cx43 exists as unopposed hemichannels that can contribute to disruption of ionic gradient and loss of essential metabolites; indeed, hemichannels have been shown to contribute to cardio- 
Table 1. Estimated percentage of phosphorylation in WT and mdx

$\begin{array}{lcc}\begin{array}{l}\text { Phosphorylation } \\ \text { site }\end{array} & \begin{array}{c}\text { Percentage of } \\ \text { phosphorylation in WT }\end{array} & \begin{array}{c}\text { Percentage of } \\ \text { phosphorylation in mdx }\end{array} \\ \text { S255 } & 0.1 \% & 16.0 \% \\ \text { S306 } & 6.5 \% & 2.8 \% \\ \text { S325, T326, S328, or S330 } & 50 \% & 22.7 \% \\ \text { S364, S365 } & 30.1 \% & 22.4 \%\end{array}$

myocyte damage and cell death in ischemic models $(16,17)$. Of note, the phosphorylation of a triplet of serine residues S325/

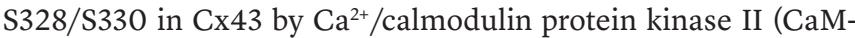
$\mathrm{KII})$ and casein kinase $1 \delta$ (CK1 $\delta$ ) has been associated with proper $\mathrm{Cx} 43$ localization to the IDs, while hypophosphorylation of this triplet leads to significant lateralization (18-20). Cx43 remodeling has been observed in numerous inherited and acquired cardiac dysfunctions, including arrhythmogenic cardiomyopathy, ischemia, and hypertrophy (11, 21-23).

In recent studies, we observed $\mathrm{Cx} 43$ protein upregulation and lateralization in human and mouse ( $\mathrm{mdx}$ ) DMD cardiac tissues (24-26). DMD heart lysates demonstrate increased levels of faster migrating, nonphosphorylated $\mathrm{Cx} 43$ isoforms on SDSPAGE, indicative of an alteration in gap junctional regulation (27). $\beta$ adrenergic stimulation by isoproterenol (Iso) administration caused severe cardiac arrhythmias and premature death in $\mathrm{mdx}$ mice, while WT mice were unaffected. The use of peptide mimetics that selectively bind to and inhibit the function of $\mathrm{Cx} 43$ hemichannels (16) protected Iso-challenged mdx mice from arrhythmogenesis and related mortality (24). Furthermore, a genetic reduction of $\mathrm{Cx} 43$ in dystrophic mice normalized cardiac $\mathrm{Cx} 43$ protein levels to those of WT, reduced $\mathrm{Cx} 43$ remodeling, and rescued the cardiac phenotype (26).

Here, we evaluated changes in phosphorylation status of $\mathrm{Cx} 43$ in both murine and human DMD hearts and discovered a substantial decrease of S325/S328/S330 phosphorylation compared with that in healthy controls. Thus, we hypothesized that the phospho-triplet is the primary player in $\mathrm{Cx} 43$ remodeling. To test our hypothesis, we generated 2 strains of compound mutant knockin mice in which the triplet of serines in $\mathrm{Cx} 43$ were replaced with either nonphosphorylatable alanines (S3A) or phospho-mimicking glutamic acids (S3E) (20) in the mdx background. We demonstrate that phospho-mimicking $\mathrm{mdx}$ mice $(\mathrm{mdxS} 3 \mathrm{E})$ were resistant to pathologic $\mathrm{Cx} 43$ remodeling, with a concomitant reduction in $\mathrm{Cx} 43$ hemichannel formation and aberrant activity. Furthermore, we uncovered a protection against augmented $\mathrm{Ca}^{2+}$ signaling during mechanical stress and excessive production of NADPH oxidase 2 (NOX2)/ROS, 2 principal DMD pathomechanisms (28), in mdxS3E hearts. We found an increase in S325/S328/S330 phosphorylation and improved gap junction retention in extracts from mdx mice with nonoxidizable CaMKII (mdx-MM:VV). This result connects ROS/oxidized CaMKII (ox-CaMKII), a promoter of $\mathrm{Ca}^{2+}$ mishandling and arrhythmias (29), with Cx43 dephosphorylation. MdxS3E mice were protected against inducible arrhythmias and displayed improved ventricular function with a reduction of cardiac fibrosis at a late stage of disease. Finally, inhibition of dystrophic microtubule (MT) cytoskeleton polymerization with colchicine (colch) resulted in enhanced Cx43 serine-triplet phosphorylation, diminished NOX2/ROS and ox-CaMKII levels, and protection against $\mathrm{Cx} 43$ remodeling, demonstrating a mechanism of $\mathrm{Cx} 43$ remodeling in dystrophic hearts. Together, our findings suggest that modulation of the $\mathrm{Cx} 43$ phosphorylation state represents what we believe to be novel therapeutic options in failing DMD hearts.

\section{Results}

Hypophosphorylation of S325/S328/S330-Cx43 in DMD hearts. We previously demonstrated that increased expression and remodeling of $\mathrm{Cx} 43$ are key contributors toward the cardiac phenotype in $\mathrm{mdx}$ mice (24-26). In the heart, decreased phosphorylation of serine residues S325/S328/S330 promotes Cx43 remodeling and arrhythmias (20,30). Thus, we investigated whether phosphorylated-S325/S328/S330 (pS-Cx43) is a critical component of Cx43 remodeling observed in $\mathrm{mdx}$ hearts. Mass spectrometry of WT and mdx mouse cardiac tissue lysates that underwent $\mathrm{Cx} 43 \mathrm{immu-}$ noprecipitation was performed to verify phosphorylation sites in a region that includes the serine triplet (Supplemental Figure 1A; supplemental material available online with this article; https:// doi.org/10.1172/JCI128190DS1). Quantification of the mass spectrometry data estimated $50 \%$ and $22.7 \% \mathrm{pS}-\mathrm{Cx} 43$ in WT and $\mathrm{mdx}$, respectively (Table 1). Next, we immunoblotted mouse ventricular tissues with the pS-Cx43 antibody and observed a considerable reduction of $\mathrm{pS}-\mathrm{Cx} 43$ levels in $\mathrm{mdx}$ tissues (relative to total $\mathrm{Cx} 43$ levels) in comparison with WT controls (47\% reduction, $P<0.001$, Figure 1A). In normal and ischemic hearts, pS-Cx43 is only detected at the ID (30). To examine $\mathrm{pS}-\mathrm{Cx} 43$ cellular localization in $\mathrm{mdx}$ tissue, we performed immunofluorescence using the pS-Cx43 antibody on mouse ventricular cryosections. In contrast with the total-Cx43 signal, which was laterally redistributed in $\mathrm{mdx}$ hearts (Figure 1B) $(24,31)$, we observed that the pS-Cx43 signal was largely confined at the ID (as shown by $\mathrm{N}$-cadherin costaining) with negligible lateralization in $\mathrm{mdx}$ hearts (Figure $1 \mathrm{C}$ ). This indicates that $\mathrm{pS}-\mathrm{Cx} 43$ is not remodeled in $\mathrm{mdx}$ hearts.

To determine whether $\mathrm{pS}-\mathrm{Cx} 43$ is also diminished in human DMD tissues, we performed immunoblotting and immunofluorescence experiments on human non-DMD and DMD ventricular tissue as described above. Consistent with our findings in mice, we measured a $79 \%$ reduction of relative $\mathrm{pS}-\mathrm{Cx} 43$ protein levels in human DMD compared with non-DMD $(P<0.01$, Figure 1D). Furthermore, we observed $\mathrm{Cx} 43$ remodeling in human $\mathrm{DMD}$ sections (Figure 1E), (24) while $\mathrm{pS}-\mathrm{Cx} 43$ was confined to the IDs (Figure 1F). Together, these results suggest that a reduction in S325/S328/S330 phosphorylation, a key mechanism of Cx43 remodeling in other cardiac pathologies, may also play an important role in DMD Cx43 remodeling.

To investigate the role of $\mathrm{pS}-\mathrm{Cx} 43$ in vivo, we crossed an established line of genetically engineered mice wherein the serine triplet was mutated to either nonphosphorylatable alanines (S3A) or phospho-mimetic glutamic acids (S3E) with WT (20) and mdx mice. Homozygous Cx43-S3A (Cx43 $\left.3^{\mathrm{S} 3 \mathrm{~A} / \mathrm{S} 3 \mathrm{~A}}\right), \mathrm{Cx} 43-\mathrm{S} 3 \mathrm{E}\left(\mathrm{Cx} 43^{\mathrm{S} 3 \mathrm{E} / \mathrm{S} 3 \mathrm{E}}\right)$, and $\mathrm{Cx} 43-\mathrm{WT}\left(\mathrm{Cx} 43^{\mathrm{WT} / \mathrm{WT}}\right)$ littermates were maintained in both WT and mdx backgrounds. Cx43-S3E mutant mice are resistant to ischemia-induced gap junctional remodeling (20). Protein extracts pre- 
A
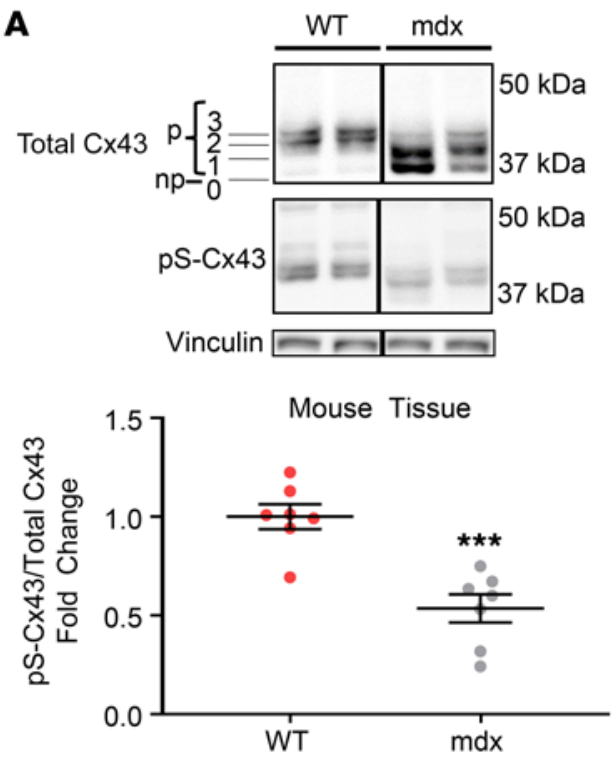

D

Total $\mathrm{Cx} 43$
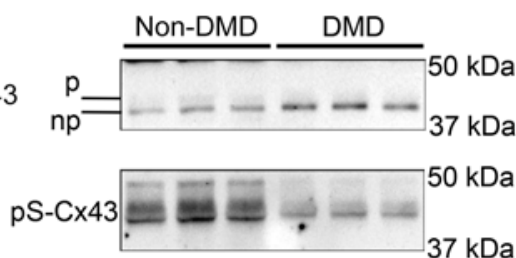

Vinculin $-\cdots-\cdots$

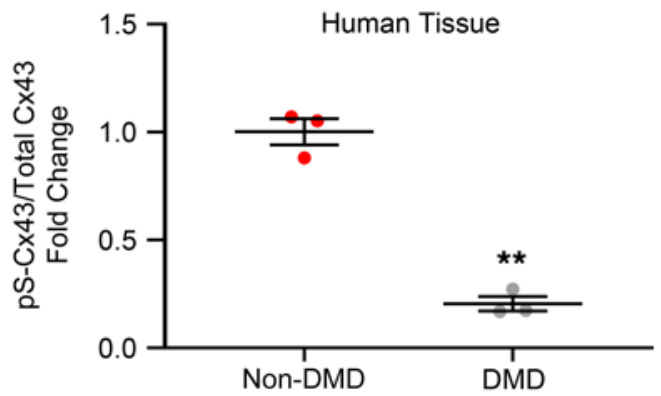

B
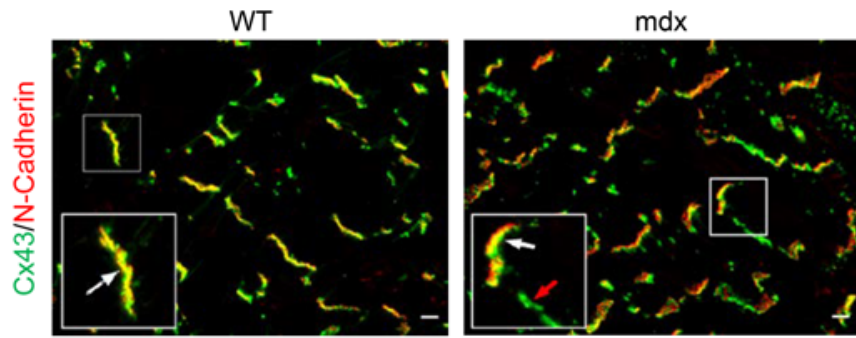

C
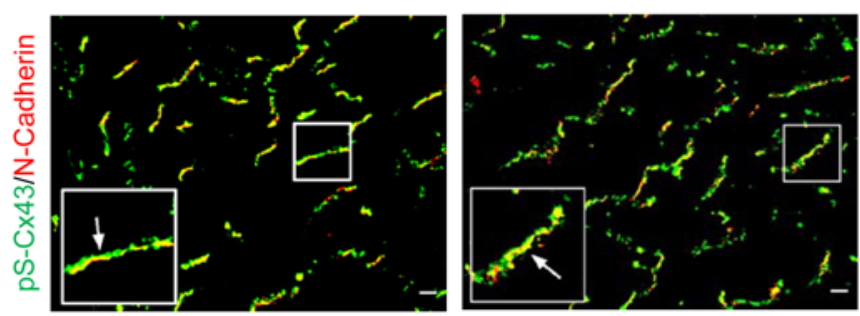

$\mathbf{E}$
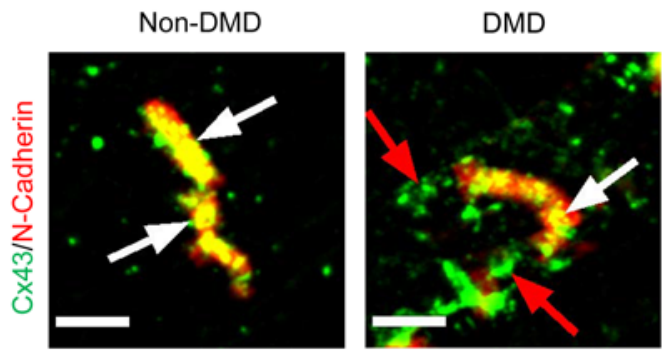

$\mathbf{F}$
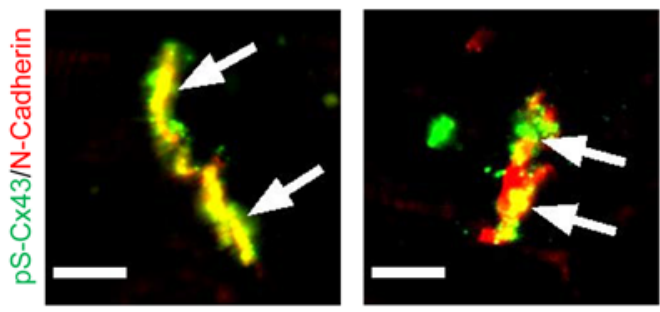

Figure 1. Phosphorylation of $\mathrm{S} 325 / \mathrm{S328} / \mathrm{S} 330$ in $\mathrm{Cx} 43$ is reduced in mouse and human dystrophic hearts. (A) Representative Western blot and quantification of WT and mdx ventricular lysates probed for pan- $\mathrm{Cx} 43$ (top), pS- $\mathrm{Cx} 43$ (middle), and vinculin (loading control, bottom). $n=7$ in both groups. ${ }^{* * *} P<$ 0.001 versus WT. Note the differential Cx43 migration patterns indicated by the phospho-isoforms P3, P2, P1, and PO in WT and mdx lysates. WT and mdx samples shown were run on the same gel, but were noncontiguous, as indicated by black lines between samples. (B) Representative immunofluorescence images (magnified images are in insets) of pan- $\mathrm{Cx} 43$ (green) and $\mathrm{N}$-cadherin (red) in 4-month-old WT and mdx ventricular cryosections. (C) Representative immunofluorescence images (magnified images are in insets) of pS-Cx43 (green) and N-cadherin (red) in 4-month-old WT and mdx ventricular cryosections. (D) Representative Western blot and quantification of human non-DMD and DMD ventricular lysates probed for pan-Cx43 (top), pS-Cx43 (middle), and vinculin (loading control, bottom). $n=3$ in both groups. ${ }^{* *} P<0.01$ versus non-DMD. p, phosphorylated isoform; np, nonphosphorylated isoform. (E) Representative immunofluorescence images of magnified IDs stained for pan-Cx43 (green) and $N$-cadherin (red) in human non-DMD and DMD ventricular cryosections. (F) Representative immunofluorescence images of magnified IDs stained for pS-CX43 (green) and N-cadherin (red) in human non-DMD and DMD ventricular cryosections. White arrows indicate $C \times 43$ localization at the IDs; red arrows indicate lateralized $C \times 43$. Data are presented as mean \pm SEM. Statistical significance was determined by 2 -sided $t$ test. Scale bars: $20 \mu \mathrm{m}$ (B and $\mathbf{C}$ ), original magnification $\times 60 ; 5 \mu \mathrm{m}(\mathbf{E}$ and $\mathbf{F})$.

pared from cardiac tissues of the phospho-mutant mice displayed changes in Cx43 protein migration on SDS-PAGE (Supplemental Figure 1B). Both WT: Cx43-S3E (WTS3E) and mdx: Cx43-S3E $(\mathrm{mdxS} 3 \mathrm{E})$ lysates displayed prominent slower-migrating P2 and P3 Cx43 phospho-isoforms with an absent PO band. The distinct phospho-isoforms represent $\mathrm{Cx} 43$ found primarily at gap junctions that participate in cellular communication $(30,32)$. Conversely,
Cx43 in WTS3A and mdxS3A lysates exhibit faster electrophoretic migration and are confined to P1 and P0 phospho-isoforms. Dystrophin was not detected in the control and phospho-mutant $\mathrm{mdx}$ hearts (Supplemental Figure 1B).

MdxS3E mice are protected from Cx43 remodeling. To determine whether remodeling was altered in our phospho-mutant mdx mice, we performed Cx43 immunohistochemistry in ventricular cryosec- 
A
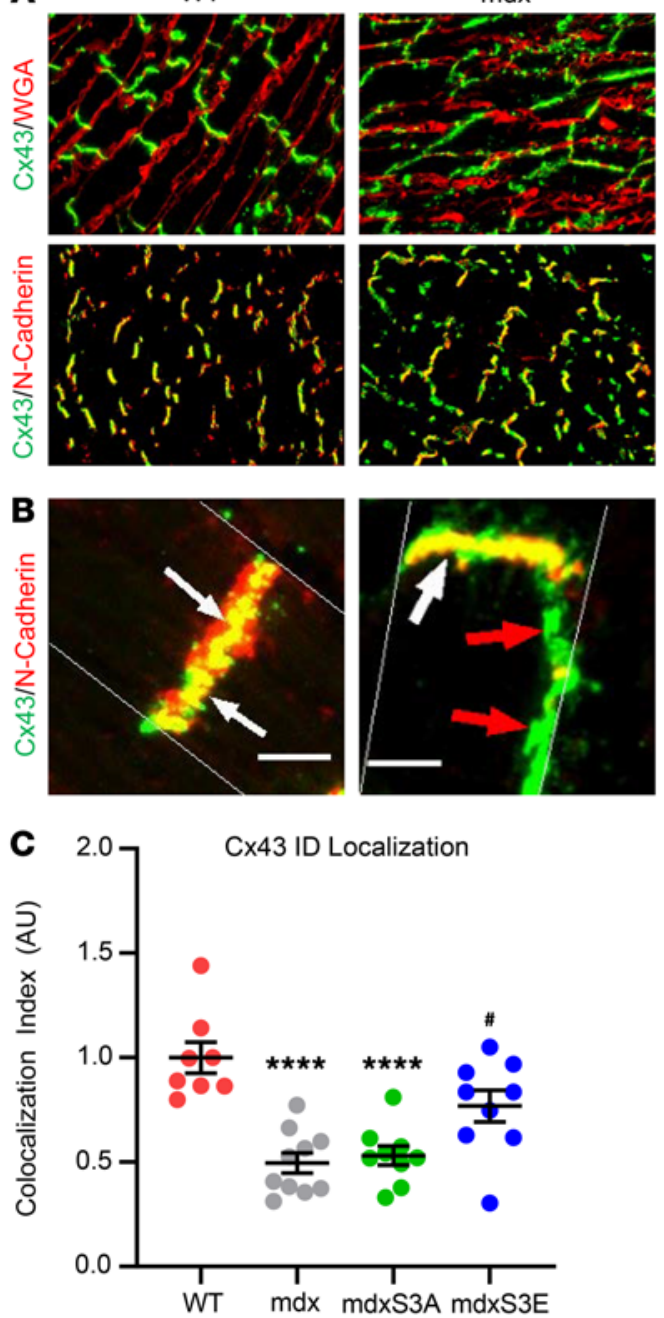

$\mathrm{mdx}$

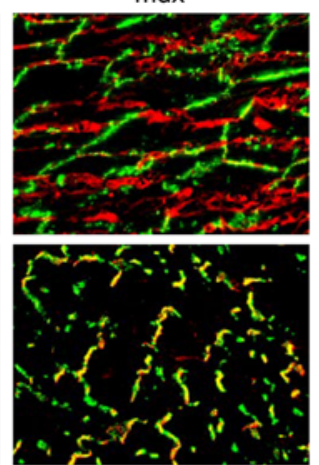

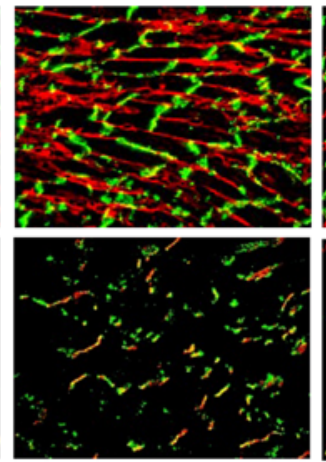

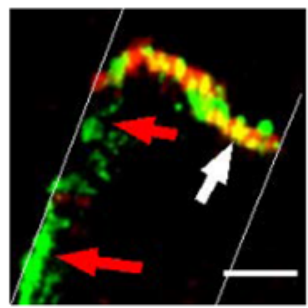

D
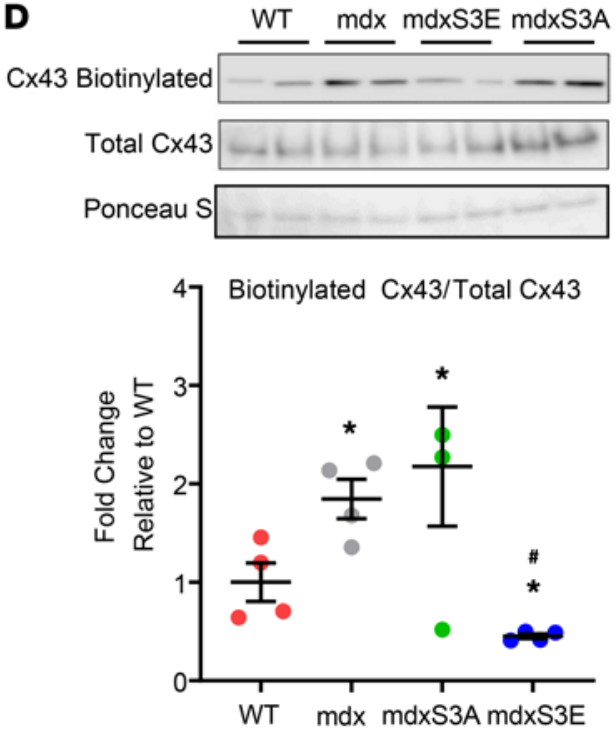

Figure 2. Cx43 remodeling is prevented in mdxS3E hearts. (A) Representative immunofluorescence images of mouse heart cryosections stained for pan- $\mathrm{C} 43$ (green) and visualized for WCA (lipid bilayer marker, red) or $\mathrm{N}$-cadherin (ID marker, red). Scale bar: $25 \mu \mathrm{m}$. (B) Representative confocal immunofluorescence images of cardiac IDs and lateral regions of cardiomyocytes stained with pan- $\mathrm{C} \times 43$ (green) and $\mathrm{N}$-cadherin (red). White arrows indicate costaining of $\mathrm{Cx} 43$ and $\mathrm{N}$-cadherin; red arrows indicate lateralized $\mathrm{Cx} 43$. Scale bars: $5 \mu \mathrm{m}$. (C) Quantification of $\mathrm{C} \times 43 / \mathrm{N}$-cadherin colocalization in confocal immunofluorescence images. All data points were normalized to the WT group mean. $n=$ 8 (WT); $n=10$ (mdx); $n=9$ (mdxS3A); $n=9$ (mdxS3E). Three to five images containing 15-20 IDs were analyzed per heart. Each dot represents a mean value per mouse. (D) Representative Western blots (top) and quantification (bottom) of $\mathrm{Cx} 43$ from biotin-perfused hearts. Top Western blot row represents biotinylated $\mathrm{C} \times 43$ protein signals, middle row represents total $C \times 43$, and bottom row represents Ponceau $S$ staining for loading. Ponceau S stain was used for loading, since we cannot detect cytosolic loading controls through biotinylation. Quantification (bottom) of biotinylated $\mathrm{Cx} 43$ levels was expressed as fold change relative to total $\mathrm{Cx} 43$ protein levels per sample. Data were normalized to WT mean group value ( $n=4$ per group). Data are presented as mean \pm SEM. ${ }^{* * * *} P<$ $0.0001 ;{ }^{*} P<0.05$ versus WT; ${ }^{\#} P<0.05$ versus $m d x$. Statistical significance determined by 1-way ANOVA followed by Tukey's post hoc test. tions. Sections from 4- to 6-month-old mice, in which we and others previously observed $\mathrm{Cx} 43$ lateralization in $\mathrm{mdx}$ hearts $(24,31)$, were stained against $\mathrm{Cx} 43$ alongside wheat germ agglutinin (WGA) (cell membrane marker) or $\mathrm{N}$-cadherin (ID marker). Wide-field and confocal immunofluorescence imaging revealed that mdxS3E hearts displayed reduction of $\mathrm{Cx} 43$ lateralization compared with mdx and mdxS3A hearts with Cx43 signal prominently confined at the IDs (Figure 2, A and B). Quantification of relative Cx43 signal found at the ID regions revealed that $\mathrm{mdxS} 3 \mathrm{E}$ mice displayed a significantly higher $\mathrm{Cx} 43$ localization at the IDs when compared with mdx and mdxS3A hearts $(P<0.05$, Figure $2 \mathrm{C})$.

To further examine $\mathrm{Cx} 43$ cellular distribution, we performed a Triton solubility assay to biochemically separate ventricular tissue into Triton X-100 insoluble and total protein fractions. In contrast with hemichannels, gap junctional plaques are insoluble to Triton X-100 (33). Western blot assays revealed that levels of junctional $\mathrm{Cx} 43$ in mdxS3E hearts were 3.6-fold higher than
$\operatorname{mdx}(P<0.05)$ and 2.8 -fold higher than $\operatorname{mdxS} 3 \mathrm{~A}(P=0.07)$ samples, reaching levels measured in WT (Supplemental Figure 2A). Next, we attempted to verify that lateral $\mathrm{Cx} 43$ molecules, seen in immunostained slides (Figure 2, A and B), represent hemichannels in the lateral myocyte membrane. To do so, we perfused isolated hearts with biotin. When perfused into an isolated heart at a reduced flow rate, membrane-impermeable biotin strongly bound to extrajunctional $\mathrm{Cx} 43$ hemichannels, thus validating it as a lateral membrane marker (Supplemental Figure 2B). After perfusion, hearts were homogenized and biotinylated proteins were pulled down with streptavidin beads, run on SDS PAGE, and probed for $\mathrm{Cx} 43$. We found a more than 4 -fold reduction of biotinylated $\mathrm{Cx} 43$ levels in mdxS3E hearts when compared with $\mathrm{mdx}$ and $\mathrm{mdxS} 3 \mathrm{~A}$ hearts $(P<0.05$, Figure $2 \mathrm{D})$. Overall, the immunofluorescence and biochemical studies confirm that substitution of Cx43-S3E is sufficient to protect dystrophic hearts from pathological $\mathrm{Cx} 43$ remodeling. 
A

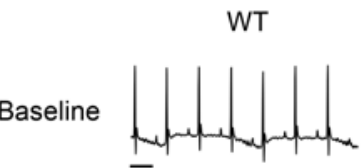

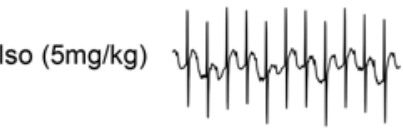

$\mathbf{C}$
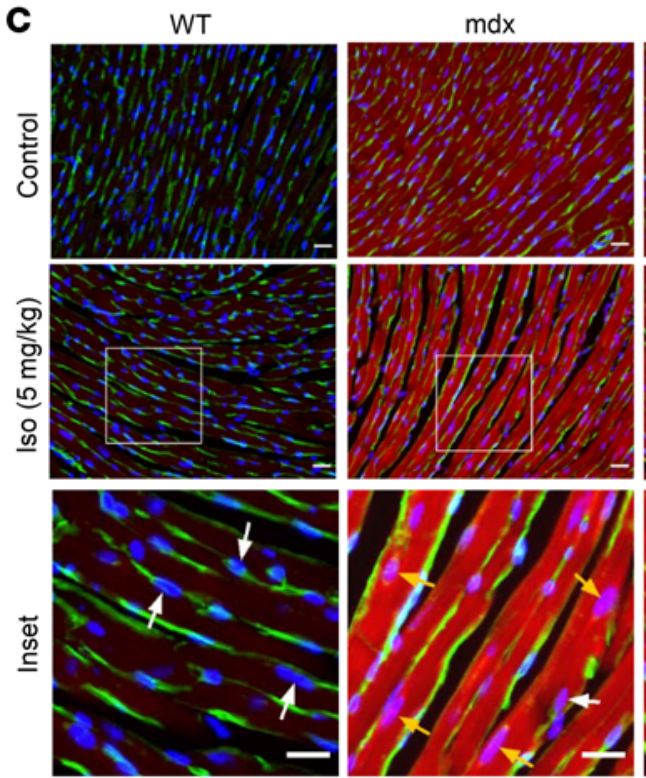

$\mathrm{mdx}$
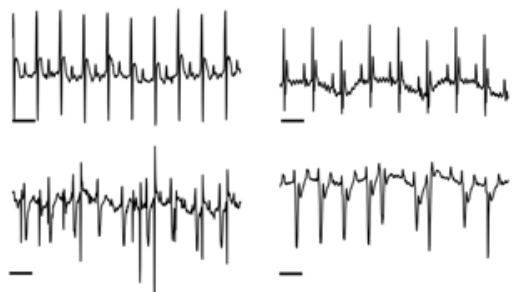

$\operatorname{mdxS} 3 \mathrm{~A}$
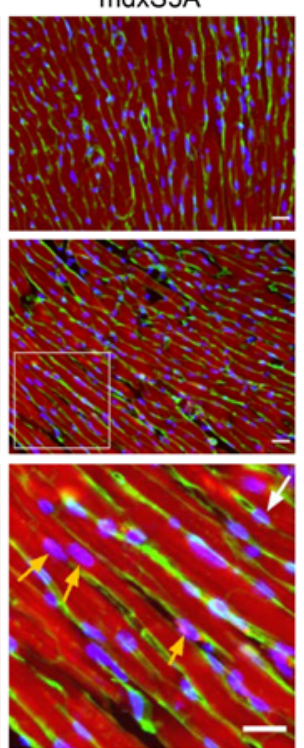

$\mathrm{mdxS} 3 \mathrm{~A}$
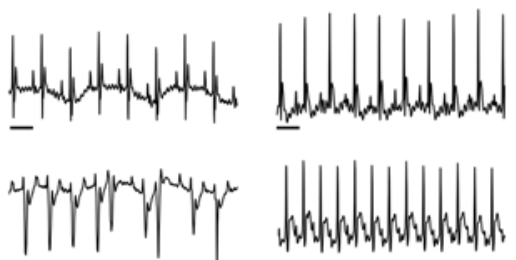

$-$

mdxS3E
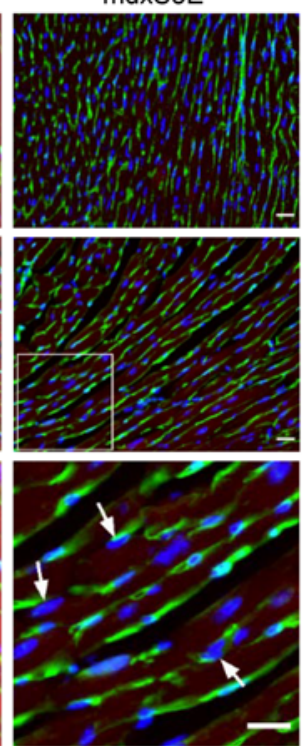

B

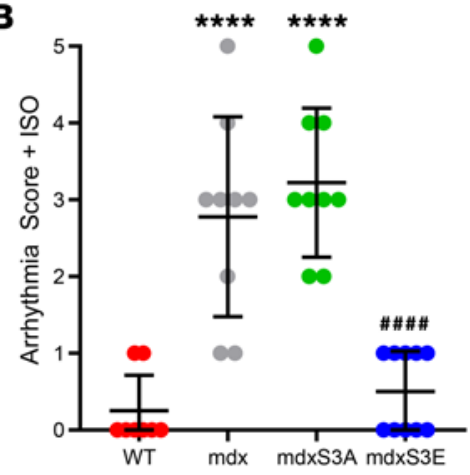

D

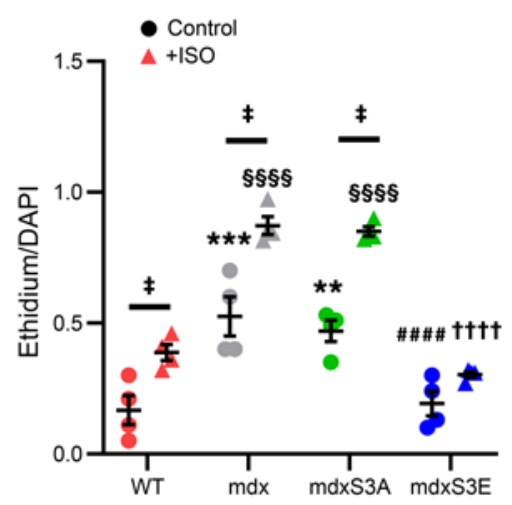

Figure 3. MdxS3E mice are protected against Iso-induced arrhythmias and aberrant Cx43 hemichannel opening. (A) Top row: representative baseline ECC recordings obtained from 4- to 6-month-old mice following anesthesia. Bottom row: representative ECG traces recorded approximately 30 minutes after Iso (5 mg/kg, i.p.) challenge. Scale bars: $100 \mathrm{~ms}$ (for all traces). (B) Arrhythmia scores based on predetermined scale where $0=$ no arrhythmias, $1=$ single PVCs, 2 = double PVCs, 3 = triple PVCs or nonsustained VT, 4 = sustained VT or AV block, $5=$ death. ${ }^{* * *} P<0.0001$ versus WT; $\# \# \# P<0.0001$ versus $\mathrm{mdx} . n=8$ (WT); $n=9$ (mdx, mdxS3A); $n=10$ (mdxS3E). (C) Representative immunofluorescence images of ethidium uptake of heart cryosections after perfusion with ethidium $(5 \mu \mathrm{m})$ under control conditions (top row) or after Iso treatment (middle row). Cryosections were visualized for ethidium (red) and stained for WCA (green) and nuclei (DAPI, blue). White boxes indicate areas magnified in the bottom row (insets). White arrows indicate nuclei that do not colocalize with ethidium; orange arrows indicate positive ethidium and nuclei colocalization. Scale bars: $20 \mu \mathrm{m}$. Original magnification $\times 20$. (D) Quantification of the dye uptake under both control (circles) and Iso (triangles) conditions. ${ }^{* *} P<0.005,{ }^{* * *} P<0.001$ versus WT control; \#\#\# $P<0.001$ versus mdx control; ${ }^{\$ \$ \$} P<0.0001$ versus WT Iso; ${ }^{t+t t} P<0.0001$ versus $\mathrm{mdx}$ Iso; ${ }^{\ddagger} P<0.05$ versus control in each genotype $(n=4$ each group per treatment). Data are presented as mean \pm SEM. Statistical significance determined by 1-way ANOVA (B) or 2-way ANOVA (D) followed by Tukey's post hoc test.

MdxS3E mice are protected from severe arrhythmias after $\beta$-adrenergic challenge. DMD patients commonly display severe ventricular arrhythmias that could contribute to sudden cardiac death (34). Adult $\mathrm{mdx}$ mice are also susceptible to arrhythmias and lethality after an acute cardiac challenge with $\beta$-adrenergic agonist Iso $(24,26,35)$. We previously demonstrated that Iso enhances $\mathrm{Cx} 43$ lateralization in vivo, suggesting that phosphorylation (or lack thereof) may also be implicated in the remodeling process under stress (24). Thus, we recorded whole animal ECGs before and after Iso challenge in 4- to 6-month-old WT, mdx, mdxS3A, and $\mathrm{mdxS} 3 \mathrm{E}$ mice to evaluate changes in ECG parameters and monitor arrhythmogenesis.

Baseline heart rate (HR) given by the RR interval was elevated in $\mathrm{mdx}$ mice $(\mathrm{RR}=126.9 \mathrm{~ms})$ compared with WT $(147.5 \mathrm{~ms}, P=$ 0.0004, Supplemental Figure 3A), in agreement with other studies (36). MdxS3A displayed the fastest baseline $\mathrm{HR}(\mathrm{RR}=122.1$ $\mathrm{ms}, P<0.0001$ versus WT), while $\mathrm{mdxS} 3 \mathrm{E}(\mathrm{RR}=138.9 \mathrm{~ms}, \mathrm{NS})$ mice exhibited a baseline HR like that of WT. Iso administration resulted in similarly elevated HRs in all mice (Supplemental Figure 3A). Baseline $\mathrm{QT}_{\mathrm{c}}$ interval was prolonged in $\mathrm{mdx}(43.8 \mathrm{~ms}, P<$ $0.0001)$ and mdxS3A (44.3 ms, $P<0.0001)$ mice compared with WT animals (WT $=26.7 \mathrm{~ms}$ ), whereas it was partially reduced in mdxS3E mice (35.7 ms) (Supplemental Figure 3B). $\mathrm{QT}_{\mathrm{C}}$ interval prolongation after Iso treatment was observed in all animals, with the longest $\mathrm{QT}_{C}$ intervals measured in $\mathrm{mdx}(74.1 \mathrm{~ms})$ and $\mathrm{mdxS} 3 \mathrm{~A}$ $(73.6 \mathrm{~ms})$ mice. $\mathrm{QT}_{\mathrm{c}}$ intervals were reduced in $\mathrm{mdxS} 3 \mathrm{E}$ mice $(58.4$ $\mathrm{ms}$ ), approaching WT values (52.8 ms) (Supplemental Figure 3B). These findings suggest that common DMD cardiac conduction deficiencies such as slowed ventricular repolarization (37) may be mitigated in $\mathrm{mdxS} 3 \mathrm{E}$ mice.

None of the mice in each group displayed arrhythmias under baseline conditions (Figure 3A). Following Iso $(5 \mathrm{mg} / \mathrm{kg}$ ) administration, both $\mathrm{mdx}$ and $\mathrm{mdxS} 3 \mathrm{~A}$ mice developed severe arrhythmias that consisted of premature ventricular contractions (PVCs), 
A
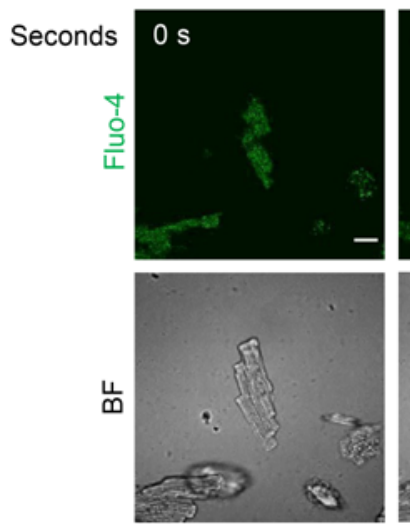

$\mathrm{mdx}$
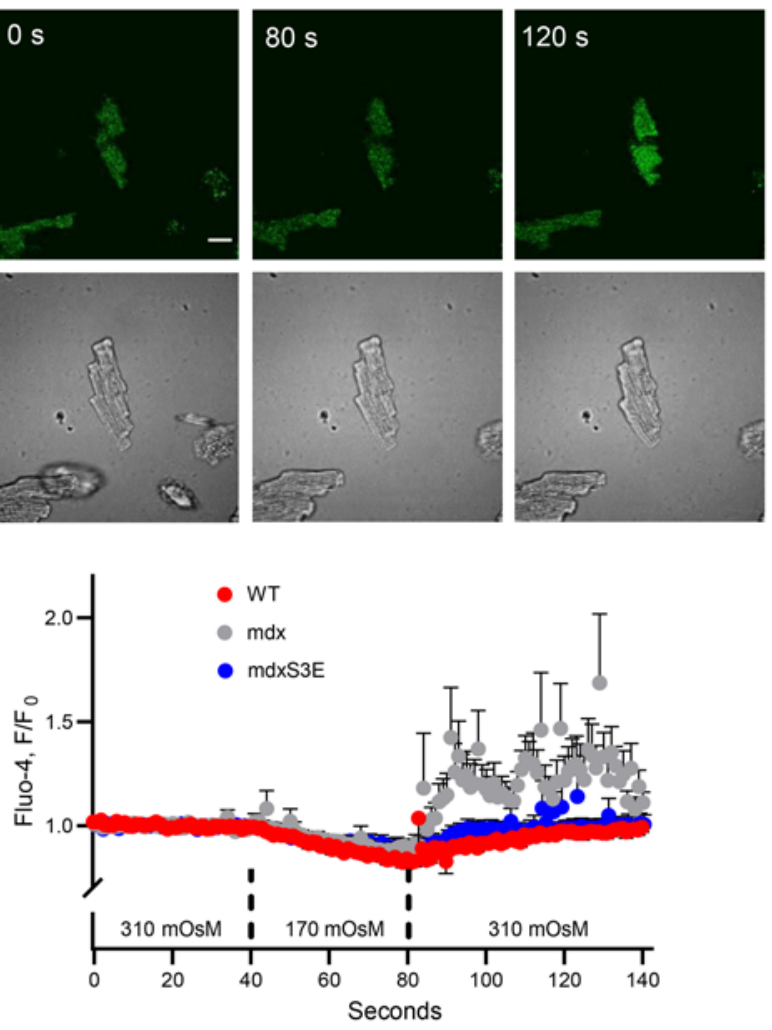

mdxS3E
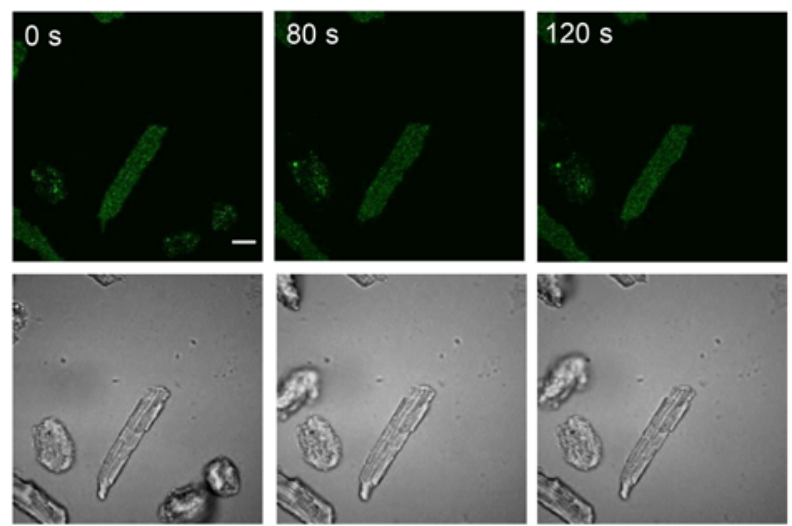

\section{B}
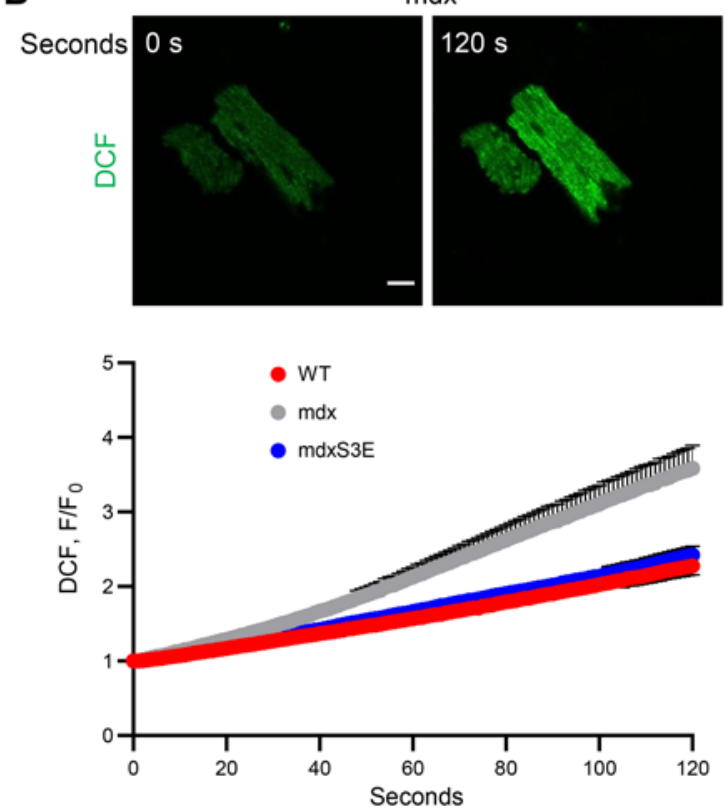

$\mathrm{mdx}$

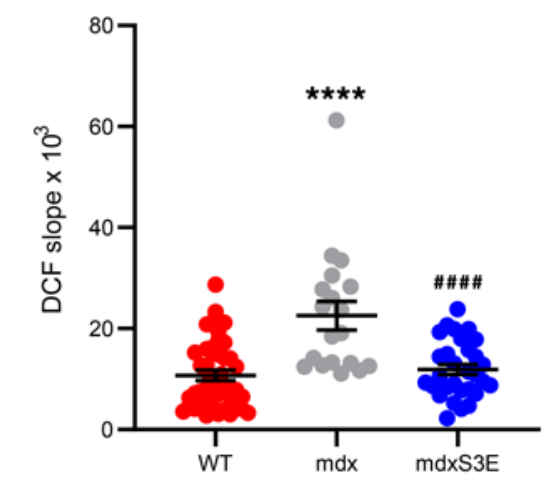

mdxS3E

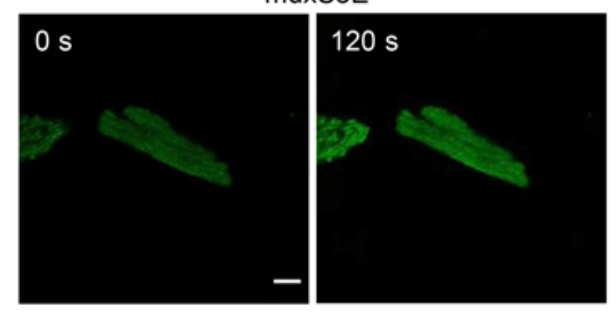

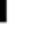

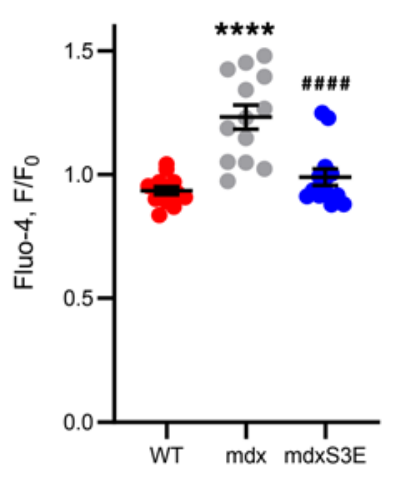


A
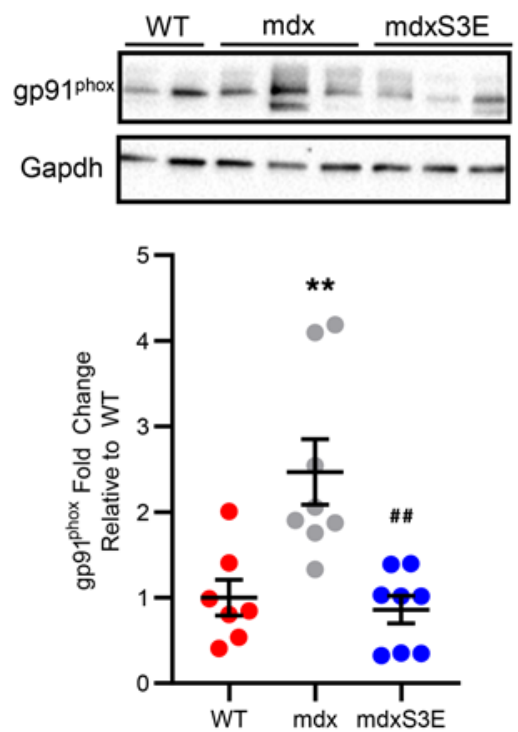

B
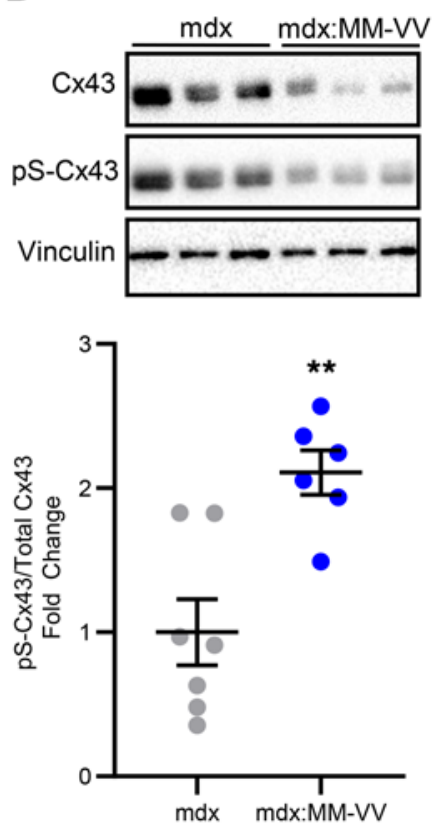

C
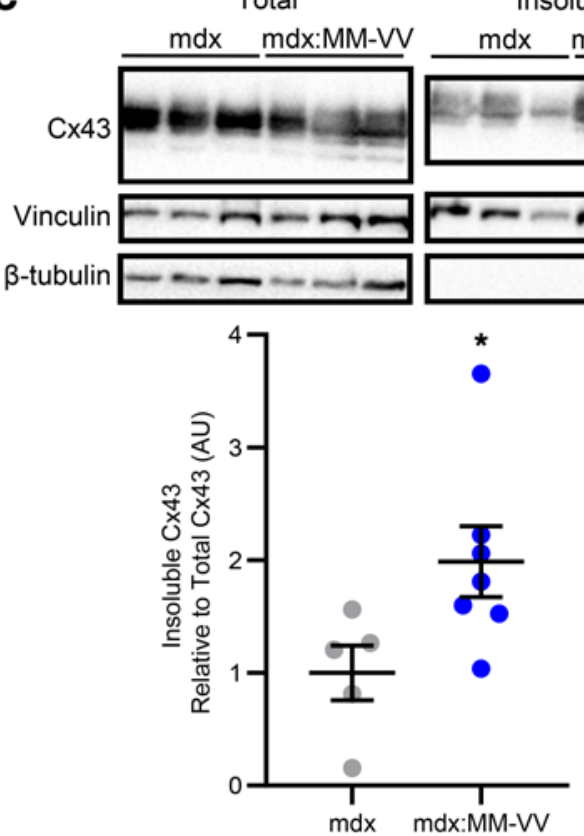

Insoluble mdx mdx:MM-VV

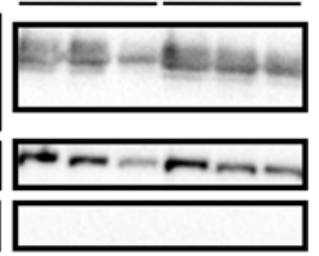

Figure 5. CaMKII oxidation leads to $\mathrm{Cx} 43$ hypophosphorylation in mdx hearts. (A) Representative Western blot (top) and quantification (bottom) for gp91 phox (NOX2 catalytic subunit) in WT (red), $\mathrm{mdx}$ (gray), and mdxS3E (blue) ventricular lysates. Gapdh was used as a loading control. ${ }^{* *} P<0.01$ versus WT; \#\# $P<0.01$ versus $\mathrm{mdx} . n=7$ (WT); $n=8$ (mdx, mdxS3E). (B) Representative Western blot (top) and quantification (bottom) of total Cx43 (top blot), pS-Cx43 (middle blot), and vinculin (bottom blot, loading control) in mdx (gray) and mdx:MM-VV (blue) whole cell lysates. $n=6$ for both genotypes. (C) Representative Western blots (left panels) and quantification (right) of Cx43 (top blots) from mdx (gray) and mdx:MM-VV (blue) heart tissue subject to Triton X-100-based tissue fractioning of insoluble (junctional, right blots) and total cell (total, left blots) lysates. Vinculin (middle blots) was used as a loading control for both fractions. $\beta$-Tubulin (bottom blots) was used as a negative control for insoluble fraction. Insoluble $\mathrm{Cx} 43$ protein levels were normalized to corresponding total $\mathrm{Cx} 43$ levels and then expressed as fold change relative to $\mathrm{mdx}$ mean value. $n=5(\mathrm{mdx}) ; n=7$ (mdx:MM-VV). ${ }^{*} P<0.05$, ${ }^{* *} P<0.01$ versus $\mathrm{mdx}$ (B and $\mathbf{C}$ ). Data are presented as mean \pm SEM. Statistical significance determined by 1-way ANOVA followed by Tukey's post hoc test (A) and 2-sided $t$ test (B and $\mathbf{C})$.

ventricular tachycardia (VT), and atrioventricular (AV) block. In contrast, WT and mdxS3E mice exhibited either single, infrequent PVCs or no abnormalities at all throughout an hour of observation (Figure $3 \mathrm{~A})$. As a result, mdxS3E mice had a markedly reduced arrhythmia score (24) (mean score $=0.5 \pm 0.17$ ) compared with $\mathrm{mdx}$ (mean score $=2.78 \pm 0.43, P<0.0001$ ) and $\mathrm{mdxS3A}$ (mean score $=3.13 \pm 0.35, P<0.0001$ ) mice (Figure 3B). Together, these results further support the importance of remodeled $\mathrm{Cx} 43$ as an arrhythmogenic substrate in a DMD animal model.

Previous findings have demonstrated that the cardiac sodium channel $\mathrm{Na}_{v} 1.5$ is downregulated and mistrafficked in ventricles of $\mathrm{mdx}^{5 \mathrm{cv}}$ mice, suggesting an additional underlying cause toward development of arrhythmias $(38,39)$. To rule out that arrhythmia protection observed in mdxS3E mice was also due to changes in $\mathrm{Na}_{\mathrm{v}} 1.5$ expression, we performed immunoblotting for $\mathrm{Na}_{\mathrm{v}} 1.5$ in $\mathrm{mdx}$ control and mutant lysates. Western blot analysis confirmed that $\mathrm{Na}_{\mathrm{v}} 1.5$ was similarly decreased in $\mathrm{mdx}(P<$ $0.05)$, mdxS3A $(P<0.01)$ and $\mathrm{mdxS} 3 \mathrm{E}(P<0.01)$ hearts when compared with WT (Supplemental Figure $3 \mathrm{C}$ ). Thus, the protein levels of $\mathrm{Na}_{\mathrm{v}} 1.5$ do not appear to contribute toward arrhythmia protection in $\mathrm{mdxS} 3 \mathrm{E}$ mice.

MdxS3E mice exhibit less aberrant hemichannel activity. Pretreatment of a selective Cx43 hemichannel blocker Gap19 protected mdx mice from Iso-induced arrhythmias (24), suggesting the important role of remodeled hemichannel activity during car- diac stress. We confirmed that a reduction of $\mathrm{Cx} 43$ lateralization via $\mathrm{Cx} 43$-S3E offered protection in vivo, but did not investigate whether there was a reduction in hemichannel activity. To investigate this, we perfused isolated hearts from control and Iso-treated mice with ethidium bromide (ethidium, $5 \mu \mathrm{M}$ ) and measured dye uptake in ventricular cryosections. Ethidium is a membrane-impermeable dye, and its uptake is mediated by $\mathrm{Cx} 43$ present in open hemichannels and not gap junctions $(40,41)$. Dye uptake in control $\mathrm{mdx}$ and $\mathrm{mdxS} 3 \mathrm{~A}$ hearts was significantly upregulated compared with WT and mdxS3E (Figure 3, C and D, control). Iso administration augmented ethidium uptake in $\mathrm{WT}$, $\mathrm{mdx}$, and mdxS3A hearts. MdxS3E heart uptake was not significantly elevated following Iso; mdxS3E hearts exhibit uptake values even less than WT hearts (Figure 3, C and D, ISO). Together, these results suggest that prevention of $\mathrm{Cx} 43$ remodeling by $\mathrm{Cx} 43-\mathrm{S} 3 \mathrm{E}$ abolishes aberrant hemichannel activity, protecting dystrophic mice from cardiac conduction abnormalities.

$M d x S 3 E$ cardiomyocytes exhibit improved calcium homeostasis and reduced ROS production. Abnormal calcium $\left(\mathrm{Ca}^{2+}\right)$ homeostasis is a prominent feature of DMD cardiomyocytes (28). Intracellular $\mathrm{Ca}^{2+}$ levels and $\mathrm{Cx} 43$ hemichannel opening are intimately linked $(42,43)$, hinting that a reduction of formation and aberrant activity of hemichannels may influence $\mathrm{Ca}^{2+}$ signaling in $\mathrm{mdx}$ cardiomyocytes, as suggested by prior findings (26). To determine the direct impact of pS-Cx43 on intracellular $\mathrm{Ca}^{2+}$ 
A

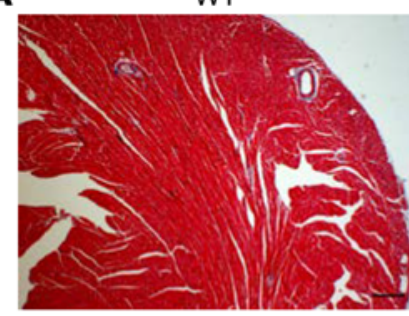

$\operatorname{mdxS} 3 \mathrm{~A}$

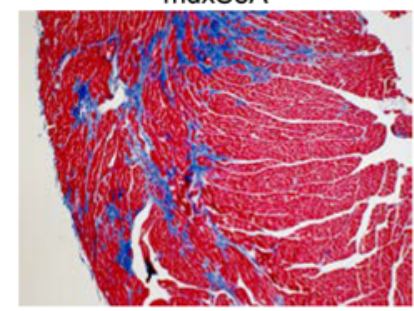

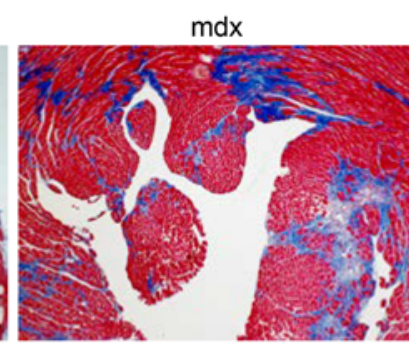

mdxS3E

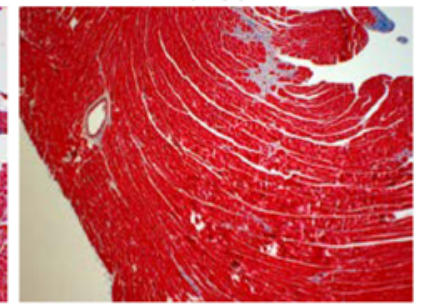

B

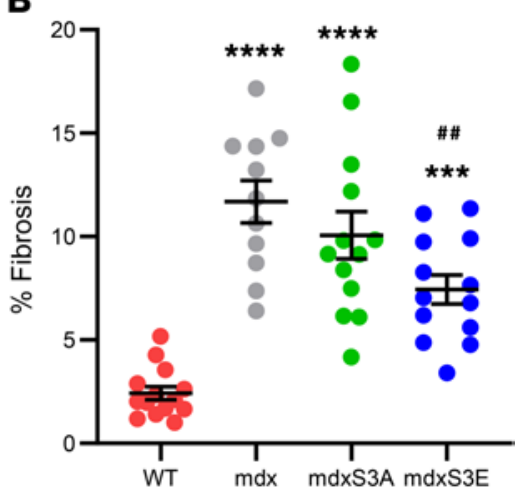

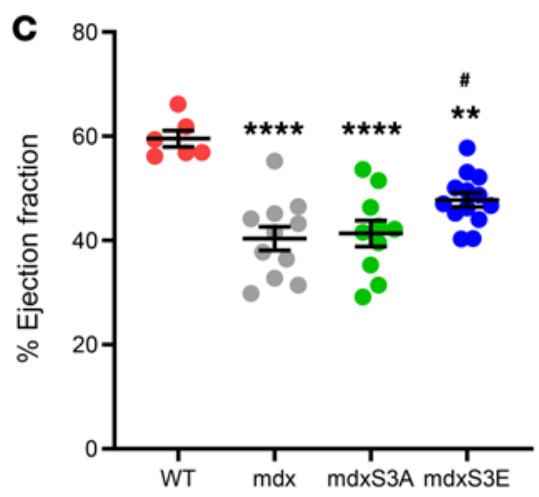

D

Survival after ISO challenge

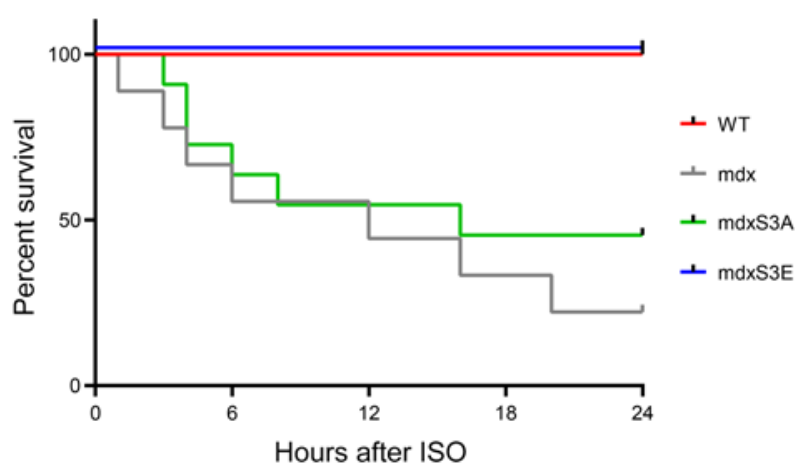

Figure 6. MdxS3E mice display long-term protection against DMD cardiomyopathy. (A) Representative images of Masson-trichrome staining on hearts of 14- to 18-month-old WT, mdx, mdxS3A, and mdxS3E mice. Red stain indicates muscle cytoplasm; blue indicates collagen deposition (fibrosis). Scale bar: $200 \mu \mathrm{m}$. (B) Quantification of fibrosis expressed as percentage of fibrotic area over total muscle area. $n=14$ (WT); $n=11$ (mdx); $n=13$ (mdxS3A); $n$ $=13$ (mdxS3E); 3-5 slices were analyzed per mouse. Each data point represents a mean value per mouse. (C) Left ventricular ejection fraction values for 14- to 18-month-old mice. $n=6$ (WT); $n=11$ (mdx); $n=10$ (mdxS3A); $n=13$ (mdxS3E). Each data point represents mean ejection fraction per mouse. (D) Kaplan-Meier survival curve for 14- to 18-month-old mice followed for 24 hours after lso ( $5 \mathrm{mg} / \mathrm{kg}$, i.p.) challenge. $n=6$ (WT); $n=9$ (mdx); $n=11$ (mdxS3A); $n=10$ (mdxS3E). ${ }^{* * *} P<0.001,{ }^{* * *} P<0.005,{ }^{* *} P<0.01$ versus $\mathrm{WT} ;{ }^{\# \# P} P 0.01,{ }^{*} P<0.05$ versus mdx. Data are presented as mean $\pm \mathrm{SEM}$. Statistical significance determined by 1-way ANOVA followed by Tukey's post hoc test (B and C).

dynamics, we isolated cardiomyocytes from approximately 3- to 4-month-old WT, mdx, and mdxS3E mice, incubated them with Fluo-4AM, and subjected them to mechanical stress through hypo-osmotic shock (44). Representative fluo-4 images of both $\mathrm{mdx}$ and $\mathrm{mdxS} 3 \mathrm{E}$ before, during, and after hypo-osmotic shock are displayed in Figure 4A. As expected, a larger intracellular $\mathrm{Ca}^{2+}$ response to hypo-osmotic shock was measured in mdx cardiomyocytes when compared with WT $(26,45)$. However, the $\mathrm{Ca}^{2+}$ stress response in $\mathrm{mdxS} 3 \mathrm{E}$ cardiomyocytes was diminished, resembling that observed in WT cardiomyocytes (Figure 4A). Average fluo4 fluorescence $\left(\mathrm{F}_{\text {ave }}\right)$ following osmotic shock relative to baseline $\left(\mathrm{F}_{0}\right)$ was quantified $\left(\mathrm{F}_{\text {ave }} / \mathrm{F}_{0}\right)$ and confirmed a significant increase in $\mathrm{mdx}$ cardiomyocytes relative to WT $(P<0.0001)$ and a reduction in mdxS3E myocytes $(P<0.0001$ relative to $\mathrm{mdx}$, Figure $4 \mathrm{~A})$.

Excessive ROS production contributes to the development of DMD cardiomyopathy $(28,46)$. Oxidative stress opens Cx 43 hemichannels $(47,48)$, while $\mathrm{Cx} 43$ gap junctions can mediate against oxidative damage (49). ROS also triggers excessive $\mathrm{Ca}^{2+}$ signaling in mdx myocytes under stress $(44,45)$. Thus, ROS production was analyzed in approximately 3- to 4-month-old WT, mdx, and $\mathrm{mdxS} 3 \mathrm{E}$ isolated cardiomyocytes loaded with a fluorescent ROS sensor, $\mathrm{H}_{2}$-DCFDA. Representative ROS production in $\mathrm{mdx}$ and mdxS3E myocytes at the beginning and ending of imaging are displayed in Figure 4B. The rate of ROS production, indicated by the increase of ROS-dependent fluorescent signal, was expectedly increased in $\mathrm{mdx}$ when compared with that in WT. However, mdxS3E myocytes displayed a significant decrease in ROS production compared with $\mathrm{mdx}(P<0.0001$, Figure $4 \mathrm{~B})$. These data imply that cardiomyocytes from mdxS3E animals exhibited a corrected cellular phenotype, like that of WT cells.

Oxidized CaMKII contributes to Cx43 hypophosphorylation. NOX2 is a major source of ROS in dystrophic tissue, particularly at early stages of disease and under acute stress conditions (28, 46). Therefore, we performed immunoblotting for gp $91^{\text {phox }}$, the 
A
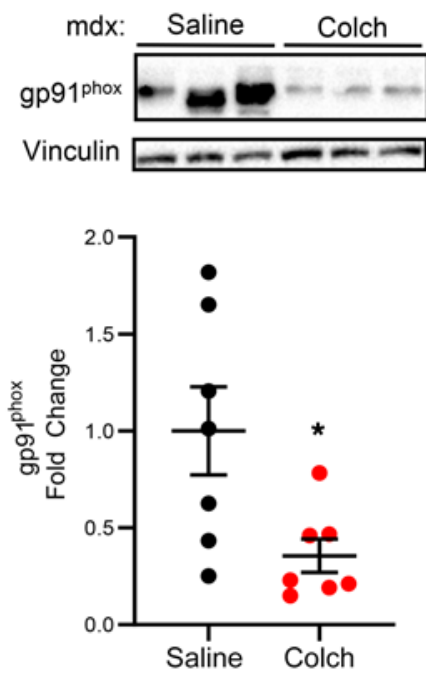

B
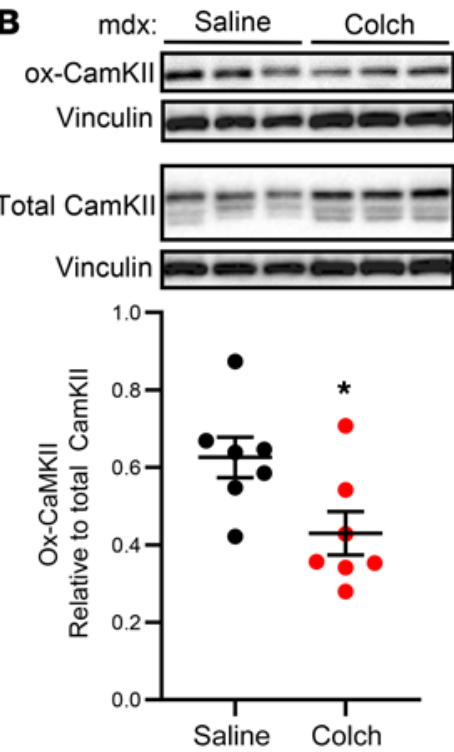

C mdx: Saline Colch
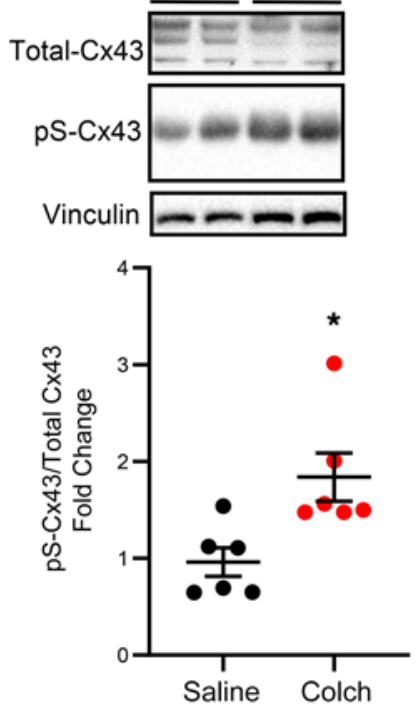

D

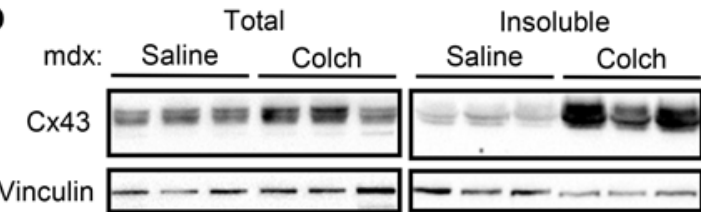

Insoluble $\mathrm{C} \times 43$

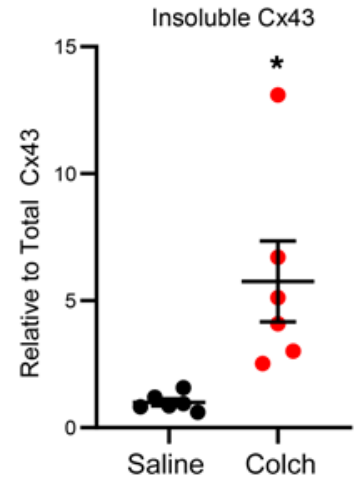

E

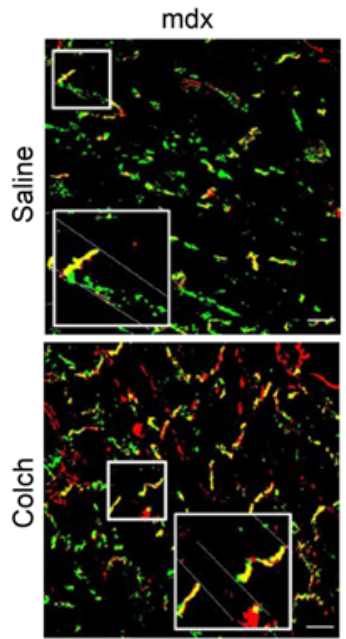

Cx43/N-Cadherin

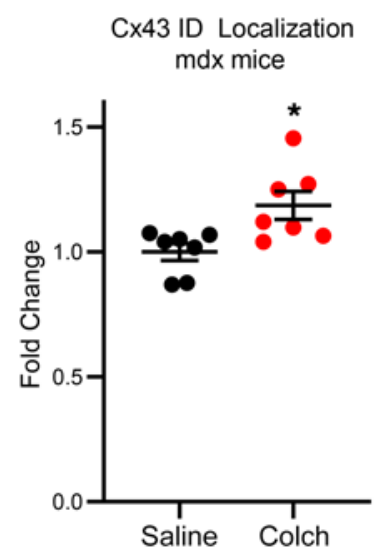

Figure 7. The dystrophic MT cytoskeleton contributes to Cx43 phosphorylation and localization. (A) Representative Western blot (top) and quantification (bottom) for $\mathrm{gp} 9$ phox $^{\text {hox }} \mathrm{mdx}$ mice treated with either saline or colch. Vinculin was used as a loading control for this and all proceeding blots. (B) Representative Western blots (top) of ox-CaMKII, (top blot), total CaMKII (bottom blots), and vinculin in mdx mice treated with either saline or colch. Ox-CaMKII protein levels were normalized to corresponding total CaMKII levels (both controlled for loading) and quantified (bottom). (C) Representative Western blots (top) for total-Cx43 (top), pS-Cx43 (middle), and vinculin (bottom) in mdx mice treated with either saline or colch. pS-Cx43 protein levels were normalized to corresponding total Cx43 levels (both controlled for loading) and quantified (bottom). (D) Representative Western blots of Cx43 and vinculin (top) from saline- or colch-treated mdx heart tissue subject to Triton X-100 based tissue fractioning. Insoluble Cx43 protein levels were normalized to corresponding total $\mathrm{Cx} 43$ levels (both controlled for loading) and then expressed as fold change relative to mdx saline mean value. (E) Left: representative confocal immunofluorescence images of $\mathrm{mdx}$ saline (top) and colch (bottom) cardiac IDs and lateral regions of cardiomyocytes stained with pan- $\mathrm{Cx} 43$ (green) and $\mathrm{N}$-cadherin (red). Scale bar: $25 \mu \mathrm{m}$. White boxes indicate areas magnified in the image; original magnification $\times 60$. Right: quantification of Cx43/N-cadherin colocalization in confocal immunofluorescence images as described for Figure 2, expressed as fold change relative to mdx saline mean value. $n=7$ per treatment ( $\mathbf{A}$ and $\mathbf{E}) ; n=6$ per treatment $(\mathbf{B}-\mathbf{D})$. ${ }^{*} P<0.05$ versus $\mathrm{mdx}$-saline. Data are presented as mean \pm SEM. Statistical significance determined by 2 -sided $t$ test (all analyses).

catalytic subunit of NOX2 (50), in left ventricular lysates of 3- to 4-month-old WT, mdx, and mdxS3E mice. Our results revealed an approximately 3 -fold decrease in $\mathrm{mdxS} 3 \mathrm{E}$ gp9 $91^{\text {phox }}$ protein levels when compared with $\mathrm{mdx}(P<0.01)$, exhibiting levels similar to those of WT (Figure 5A). This finding suggests that a reduction of ROS production observed in mdxS3E myocytes (Figure 4B) may implicate a reduction of cardiac NOX2.
Aberrant activity of CaMKII by NOX2/ROS oxidation promotes $\mathrm{Ca}^{2+}$ mishandling and arrhythmias in mdx mice (51-53). Inhibition of ox-CaMKII by knockin replacement of regulatory domain methionines with valines (MM-VV [CaMKII M281/282V]) normalized $\mathrm{Ca}^{2+}$ behavior and prevented tachycardia in mdx mice (mdx:MM-VV) (53). Of interest, CaMKII also phosphorylates Cx43-serines 325/328/330 (19), which suggests that exacerbated 
CaMKII activity may alter pS-Cx43 in DMD hearts. To investigate this, we performed immunoblotting for $\mathrm{Cx} 43$ and $\mathrm{pS}-\mathrm{Cx} 43$ in approximately 4- to 6-month-old mdx and mdx:MM-VV whole cell lysates. We found a 2 -fold increase in relative $\mathrm{pS}-\mathrm{Cx} 43$ protein levels in $\mathrm{mdx}: \mathrm{MM}-\mathrm{VV}$ lysates when compared with $\mathrm{mdx}(P<0.01$, Figure 5B). Furthermore, we conducted a Triton solubility assay in mdx:MM-VV tissues to assess for levels of junctional $\mathrm{Cx} 43$ in comparison with $\mathrm{mdx}$. Accordingly, we found a 2 -fold increase of Triton insoluble Cx43 in mdx:MM-VV extracts $(P<0.05$, Figure $5 \mathrm{C}$ ). These findings indicate that increased ox-CaMKII in an oxidative DMD environment decreases $\mathrm{pS}-\mathrm{Cx} 43$, but this can be blunted by preventing the oxidation of CaMKII. In addition, mimicking $\mathrm{pS}-\mathrm{Cx} 43$ can reduce excessive ROS production and normalize $\mathrm{Ca}^{2+}$ homeostasis in DMD hearts (Figure 4).

Aged mdxS3E mice exhibit improved cardiac function and morphology. We have investigated the role of $\mathrm{pS}-\mathrm{Cx} 43$ in young adult (4 to 6 months old) mdx mice, months before the development of dilated cardiomyopathy known to occur at approximately 10 months $(25,54)$. We next assessed pertinent cardiovascular parameters in aged (14 to 18 months old) mice. Aged ventricular tissues were assessed for histopathology by Masson's trichrome staining (Figure 6A). WT animals developed negligible cardiac fibrosis, while all animals with $\mathrm{mdx}$ background displayed enhanced fibrosis (Figure 6A). However, among the mdx groups, a substantially lower degree of fibrosis was measured in $\operatorname{mdxS} 3 \mathrm{E}(7.44 \%, P<0.01)$ hearts $(\mathrm{mdx}=11.68 \%, \mathrm{mdxS} 3 \mathrm{~A}=10.07 \%$, Figure $6 \mathrm{~B})$.

To analyze changes in overall cardiac function, we performed transthoracic echocardiography in aged mice. Left ventricular ejection fraction improved in mdxS3E (47.8\%) compared with both $\operatorname{mdxS} 3 \mathrm{~A}(41.35 \%)$ and $\mathrm{mdx}(40.37 \%, P<0.05)$ mice (Figure $6 \mathrm{C})$. Consistent with our observations in young mice, both aged $\mathrm{mdx}$ and mdxS3A mice developed severe arrhythmias within minutes after Iso challenge, while $\mathrm{mdxS} 3 \mathrm{E}$ mice were largely protected throughout the duration of analysis (Supplemental Figure 4A). Upon completion of ECG monitoring, mice were then followed up to 24 hours after Iso challenge to assess mortality. A majority of young adult mdx mice die within 24 hours of Iso administration (24). All mdxS3E mice survived in the 24 hours following Iso challenge, while only $22 \%$ of $\mathrm{mdx}$ mice survived (Figure 6D). Unexpectedly, we also observed a modest survival benefit in mdxS3A ( 45\%) mice following Iso.

To rule out that the survival benefit attributed to $\mathrm{mdxS} 3 \mathrm{E}$ mice was due to indirect improvements in respiratory function, we performed diaphragm ultrasonography (55). The amplitude of diaphragm movement during inspiration declined significantly in all $\mathrm{mdx}(\mathrm{mdx}=0.33 \mathrm{~mm}, \mathrm{mdxS} 3 \mathrm{~A}=0.33 \mathrm{~mm}, \mathrm{mdxS} 3 \mathrm{E}=0.35 \mathrm{~mm})$ genotypes compared with WT (all $P<0.01$, WT $=0.56 \mathrm{~mm}$ ), with no evident improvement in mdxS3E mice (Supplemental Figure 4B). Next, we assessed fibrotic damage, which is a major contributor to cardiorespiratory failure in $\mathrm{mdx}$ mice $(56,57)$. In contrast to what occurred in the heart, Masson's trichrome staining revealed that $\mathrm{mdxS} 3 \mathrm{E}$ diaphragms were visibly fibrotic, resembling those of mdx and mdxS3A. Quantification of diaphragm fibrotic areas confirmed that mdxS3E diaphragms develop fibrosis (40.82\%) as its $m d x$ counterparts $(\mathrm{mdx}=32 \%, \mathrm{mdxS} 3 \mathrm{~A} 41.23 \%$, Supplemental Figure $4 \mathrm{C}$ ). Taken together, the functional and histopathological data both indicate that the respiratory system does not play a role in the rescue of cardiomyopathy in mdxS3E mice.
The dystrophic MT cytoskeleton contributes to CX43 triplet phosphorylation and remodeling. Growing evidence indicates that the MT cytoskeleton plays an essential role in the development of DMD cardiomyopathy. The dense and disorganized MT network (58) in mdx cardiomyocytes correlates strongly with enhanced ROS production and $\mathrm{Ca}^{2+}$ mishandling (59-62). Targeting the MT network protects mdx mice against stress-induced arrhythmias (35). Importantly, intracellular trafficking of $\mathrm{Cx} 43$ largely depends on MTs (63). MT-dependent forward trafficking of $\mathrm{Cx} 43$ to the IDs is disrupted in stressed cardiomyocytes, yielding less junctional $\mathrm{Cx} 43$ and impaired gap junctional function (64). It has also been suggested that $\mathrm{Cx} 43$ remodeling in diseased hearts can be explained by redirectionality of MTs toward the lateral membrane in cardiomyocytes (65).

Therefore, to examine a potential causal relationship between MTs and Cx43 remodeling in mdx mice, we implanted 3-monthold mdx mice with miniosmotic pumps administering either saline or colch, a known MT depolymerizer with protective effects in mdx mice $(66,67)(0.4 \mathrm{mg} / \mathrm{kg} /$ day $)$, for 4 weeks. Ventricular tissue was collected at the conclusion of the treatment. Reduction of MT density with colch treatment was confirmed via a reduction in $\beta$-tubulin at both the tissue protein level and fluorescent signal in isolated cardiomyocytes (Supplemental Figure 5, A and $\mathrm{B})$. NOX2/gp91 ${ }^{\text {phox }}$ protein levels significantly decreased by $65 \%$ in colch-treated $\mathrm{mdx}$ ventricles $(P<0.05$, Figure $7 \mathrm{~A})$. Furthermore, immunoblotting revealed a $31 \%$ reduction in relative ox-CaMKII levels in colch-treated hearts $(P<0.05$, Figure $7 \mathrm{~B})$. We next assessed for changes in $\mathrm{Cx} 43$ wherein we detected a 2-fold increase in relative $\mathrm{pS}-\mathrm{Cx} 43$ levels in colch-treated $\mathrm{mdx}$ lysates compared with saline $(P<0.05$, Figure $7 C)$. Next, we examined cellular distribution of $\mathrm{Cx} 43$ in a Triton solubility assay. We measured a near 6-fold increase in insoluble $\mathrm{Cx} 43$ in colch-treated mdx hearts $(P<0.05$, Figure 7D). Driven by these findings, we performed $\mathrm{Cx} 43$ immunohistochemistry in ventricular cryosections. Confocal immunofluorescence imaging revealed a reduction of $\mathrm{Cx} 43$ remodeling in colch-treated hearts (Figure 7E), resembling what was previously seen in mdxS3E hearts (Figure 2). Quantification of relative $\mathrm{Cx} 43$ signal found at the IDs confirmed a significantly higher $\mathrm{Cx} 43$ localization to the IDs in colch-treated $\mathrm{mdx}$ hearts $(P<0.05$, Figure $7 \mathrm{E})$.

\section{Discussion}

In previous studies, we provided evidence supporting the role of $\mathrm{Cx} 43$ remodeling in the development of DMD cardiomyopathy, but were unable to establish a mechanistic explanation (24-26). In this study, mdx mice with a substitution of phospho-mimicking glutamic acids at serines 325/328/330 in Cx43 (mdxS3E) were protected against remodeling and displayed a drastic reduction in hemichannel formation (Figure 2) and activity in vivo (Figure 3). Furthermore, both ROS production and intracellular $\mathrm{Ca}^{2+}$ homeostasis were normalized in mdxS3E cardiomyocytes (Figure 4). We also found that high levels of ox-CaMKII were associated with hypophosphorylation of the $\mathrm{Cx} 43$ serine-triplet and reduced $\mathrm{Cx} 43$ formation at the gap junctions in dystrophic hearts (Figure 5). Because of the chronic remodeling protection in vivo, mdxS3E mice were protected against Iso-induced arrhythmias and lethality (Figure 3) as well as the development of late-stage 
cardiomyopathy (Figure 6). Finally, our data suggest an important contributor toward $\mathrm{Cx} 43$ remodeling in DMD hearts - the MT cytoskeleton. Targeting the densely disorganized, dystrophic MTs in vivo with colch reduced ROS production concomitant with ox-CaMKII levels, enhanced pS-Cx43, and importantly, provided protection against cardiac $\mathrm{Cx} 43$ remodeling (Figure 7). Overall, our findings suggest a detailed interplay between $\mathrm{Cx} 43$ remodeling and DMD cardiomyopathy.

Mimicking Cx43 phosphorylation was sufficient to correct several abnormalities prominent in dystrophic cardiomyocytes. Our results are consistent with prior studies in which either mimicking or enhancement of $\mathrm{pS}-\mathrm{Cx} 43$ conferred cardioprotection in mouse models of hypertrophy and transverse aortic constriction (TAC), respectively $(18,20)$. pS-Cx43 also preserved gap junctional communication in cardiomyocytes unresponsive to FGF, an important mediator of cardioprotection (68). Wang et al. recently reported that injection of AAV9 carrying Cx43-S3E into post-myocardial infarction hearts improved cardiac function and promoted adult cardiomyocyte redifferentiation at the site of infarct (69). This discovery may provide a missing link between $\mathrm{Cx} 43$ phosphorylation and the protection against cardiomyocyte cell death and fibrosis, hallmark features of DMD cardiomyopathy that were mitigated in $\mathrm{mdxS} 3 \mathrm{E}$ mice.

To our knowledge, we are the first to establish a mechanistic model describing $\mathrm{Cx} 43$ remodeling in dystrophic cardiomyocytes. We propose that excessive ROS production via hyperdensification of MTs in dystrophic hearts (59) promotes excessive oxidation of CaMKII (53) that leads to hypophosphorylation of S325/S328/S330. Reduced phosphorylation subsequently leads to pathological $\mathrm{Cx} 43$ lateralization and excessive $\mathrm{Cx} 43$ hemichannel activity, increasing susceptibility to stress-induced arrhythmias and development of cardiomyopathy. The genetic correction of $\mathrm{Cx} 43$ remodeling with $\mathrm{Cx} 43$-S3E normalizes the oxidative environment and $\mathrm{Ca}^{2+}$ homeostasis and provides protection against stress-induced arrhythmias and cardiac disease. However, our proposed model does not preclude the possibility of other important $\mathrm{Cx} 43$ protein interactions or modifications. Specifically, the Shaw laboratory has proposed that filamentous actin (f-actin) acts as a critical Cx43 vesicle delivery system. They suggest that stabilized actin filaments guide growing MTs toward the cell surface, increasing delivery of $\mathrm{Cx} 43$ to IDs $(70,71)$. Dystrophin binds to actin and affects actin cytoskeleton dynamics (72, 73), hinting that the MT/actin relationship may also be altered in dystrophic hearts with and without MT inhibition. Furthermore, phosphorylation of Y247 on Cx43 by Src-kinase inhibits the binding of $\mathrm{Cx} 43$ to tubulin (74), which has been suggested to increase Cx43 disassembly and reduce cellular communication (75). Src kinase can be activated by ROS, and its levels are increased in mdx muscles (76). Thus, Src may also be altered by inhibition of dystrophic MTs much like CaMKII. Nevertheless, we provide strong evidence for the MT cytoskeleton as a mediator between dystrophin loss and $\mathrm{Cx} 43$ remodeling, a previously unestablished relationship in DMD hearts.

In this study, we uncover S325/S328/S330 hypophosphorylation as a harmful consequence of cardiac CaMKII oxidation. Since S325/S328/S330 can be directly phosphorylated by CaMKII (19) and oxidation of CaMKII typically leads to hyperphosphor- ylation of its targets (29), one may suspect that oxidative activation of CaMKII enhances pS-Cx43. However, cardiac extracts from mdx mice with nonoxidizable CaMKII (mdx:MM-VV) (53) displayed increases in relative $\mathrm{pS}-\mathrm{Cx} 43$ levels when compared with $\mathrm{mdx}$ (Figure 5B), a counterintuitive result in need of further investigation. To this end, we analyzed the phosphorylation status of Cx43-S365, another site that is both hypophosphorylated in mdx hearts (Table 1) and is a target of CaMKII phosphorylation (19). However, we found no difference in the level of phosphorylated Cx43-S365 compared with total Cx43 between $\mathrm{mdx}$ and mdx:MM-VV heart lysates (Supplemental Figure 6). Cx43 can be phosphorylated at more than 20 different residues by different kinases, and at least 9 of the phosphorylated residues have been reported to be phosphorylated by 2 to 4 different kinases (14). We believe that a potential crosstalk between protein kinases may obscure some of their individual contributions. Future studies in which additional kinases are mutated in $\mathrm{mdx}$ hearts will need to be performed in order to unmask the role of ox-CaMKII in specific sites of $\mathrm{Cx} 43$. Nevertheless, prevention of oxidation of CaMKII by MM-VV mutation enhances gap junctional $\mathrm{Cx} 43$ in mdx mice principally via $\mathrm{pS}-\mathrm{Cx} 43$.

It is important to note that, although the focus of this study has been on $\mathrm{Cx} 43$ remodeling, the cardioprotection observed in mdxS3E mice may also be attributed to enhanced gap junctional intercellular communication (GJIC). In most cardiac disease models in which gap junctional remodeling is observed, $\mathrm{Cx} 43$ is not only laterally redistributed, but its expression levels are significantly reduced at the ID (22). This reduction causes gap junctional uncoupling, which leads to slowed conduction and discontinuous propagation, 2 key arrhythmic substrates in multiple animal models (11). In fact, most therapeutic strategies targeting $\mathrm{Cx} 43$ focus on preservation of $\mathrm{Cx} 43$ at the ID to promote GJIC rather than prevention of remodeling (77-79). Our data showing enhanced $\mathrm{Cx} 43$ in the triton-insoluble, gap junctional protein fractions of mdxS3E hearts (Supplemental Figure 2A), as well as increased relative $\mathrm{Cx} 43$ signal found at the ID regions in cryosections of mdxS3E hearts (Figure 2), suggest enhanced Cx43 localization at the ID. However, correction of cardiac disease in dystrophic mice can be achieved without a full restoration of ID-associated $\mathrm{Cx} 43$ (26). This supports the notion that remodeling but not reduced ID-Cx43 is the key pathological event in dystrophic hearts.

Despite a global genetic knockin, the dystrophic phenotype is only rescued in the heart (Figure 6) and not in the surrounding skeletal muscles, as evidenced by the weakened and visibly fibrotic diaphragm in aged mdxS3E mice (Supplemental Figure 4, B and C). Cardiac and respiratory dysfunctions coexist in late-stage DMD patients, with one negatively affecting the other (80). As opposed to cardiac muscle, adult skeletal muscle does not require gap junctions to coordinate muscle contraction (81). However, de novo expression of $\mathrm{Cx} 43$ hemichannels contributes toward muscle degeneration in denervated dystrophic fast skeletal muscle fibers, such as the diaphragm (82-84). Thus, Cx43 hemichannels were suggested to be a viable therapeutic target for dystrophic muscle. Nevertheless, our results support the notion that correction of dystrophic cardiomyopathy by suppression of $\mathrm{Cx} 43$ remodeling can proceed independently of the presence of dystrophic respiratory muscles. 
There are some limitations in the present report. First, we did not detect an overt exacerbation of the dystrophic cardiac phenotype in mdxS3A mice as we had originally expected. In fact, we saw a modest survival benefit in aged $\mathrm{mdxS} 3 \mathrm{~A}$ mice following Iso challenge when compared with mdx mice (Figure 4D). At this point, we can rule out any variations in background, since only mdx control and mutant littermates were used in our experiments (see Methods). However, there may be compensatory mechanisms that are specifically triggered in the $\mathrm{mdxS} 3 \mathrm{~A}$, but not $\mathrm{mdx}$, hearts that mitigate the loss of phosphorylation at $\mathrm{Cx} 43$ sites 325/328/330 (85). Second, we did not address whether enhanced densification of mdx MTs exacerbated Cx43 remodeling. Counter to our model, a previous report demonstrated that increasing acetylated $\alpha$-tubulin levels and stabilizing MTs via paclitaxel (Taxol) treatment in mice with lamin $\mathrm{A} / \mathrm{C}$ dilated cardiomyopathy prevented mislocalization of $\mathrm{Cx} 43$ and improved ventricular conduction (86). However, we must point out that Taxol treatment also increases levels of detyrosinated tubulin, which is detrimental when enhanced in DMD and other cardiac disorders $(58,59,87)$. Therefore, further stabilization of dystrophic MTs will not only exacerbate the DMD phenotype through previously defined mechanisms, such as impaired contractility (35), increased ROS production (62), and aberrant $\mathrm{Ca}^{2+}$ signaling (61), but may also enhance $\mathrm{Cx} 43$ mislocalization.

The clinical potential for targeting $\mathrm{Cx} 43$ in the treatment of DMD cardiomyopathy is strengthened by the growing number of reported Cx43-based therapeutics approaches for treatment of cardiac pathologies. The injection of muscle-derived stem/progenitor cells with overexpression of $\mathrm{Cx} 43$ into patients with heart failure improved myocardial viability in a phase 1 clinical study (88). Our present study along with our earlier reports illustrate the importance of lateral Cx43 hemichannel activity as a principal contributor to the arrhythmogenic phenotype in DMD mice (Figure 3) (24). Nonapeptide Gap19 that selectively inhibits Cx43 hemichannels counteracted the deleterious effects of myocardial ischemia/reperfusion injury $(16,17)$. Antagonism of aldosterone, a well-known contributor toward heart failure, enhances $\mathrm{pS} 325-\mathrm{Cx} 43$ phosphorylation and reverse remodeling in diseased mice (18). Interestingly, the recent inclusion of aldosterone antagonist eplerenone in early DMD cardiomyopathy treatments delayed progressive cardiac dysfunction when compared with standard therapy (89). Eplerenone's direct impact on $\mathrm{Cx} 43$ is unknown, but evidence suggests that it may enhance phosphorylation and improve $\mathrm{Cx} 43$ function through the nitric oxide (NO) pathway (90), a key mediator of $\mathrm{Cx} 43$ localization in hypertrophic hearts (91).

Collectively, the present study provides evidence that posttranslational phosphorylation of S325/S328/S330 in Cx43 is a key event that protects against gap junctional remodeling and the development of cardiomyopathy in the DMD setting. This study provides insight into how dystrophic pathomechanisms intersect with $\mathrm{Cx} 43$ phosphorylation, localization, and function in the heart. Our results, coupled with the extensive knowledge and potential ascribed to $\mathrm{Cx} 43$ therapeutics, further strengthens the potential for $\mathrm{Cx} 43$-based strategies to combat DMD-cardiomyopathy.

\section{Methods}

Detailed descriptions of the experimental methods are provided in the Supplemental Methods.
Mouse studies. Heterozygous WT (C57BL/6) knockin Cx43 $3^{\mathrm{S3A} / \mathrm{WT}}$ and $\mathrm{Cx} 43^{\mathrm{SBE} / \mathrm{WT}}$ mice were provided in-house. These were bred to generate homozygous $\mathrm{Cx} 43^{\mathrm{WT} / \mathrm{WT}}$ (WT), $\mathrm{Cx} 43^{\mathrm{S3A} / \mathrm{S3A}}$ (WTS3A), and $\mathrm{Cx} 43^{\mathrm{S3E} / \mathrm{S3E}}$ (WTS3E) mice (20). To generate Cx43-mutant DMD mice, mdx (C57BL/10ScSn-DMD ${ }^{\mathrm{mdx}} / \mathrm{J}$ ) mice were crossed with both WTS3A and WTS3E mice until mdx: $\mathrm{Cx} 43^{\mathrm{S3A} / \mathrm{WT}}$ and $\mathrm{mdx}: \mathrm{Cx} 43^{\mathrm{SBE} / \mathrm{WT}}$ heterozygous mice were produced. These mice were then bred to generate homozygous mdx: $\mathrm{Cx} 43^{\mathrm{WT} / \mathrm{WT}}(\mathrm{mdx}), \mathrm{mdx}$ : $\mathrm{Cx} 43^{\mathrm{SBA} / \mathrm{S3A}}(\mathrm{mdxS} 3 \mathrm{~A})$, and $\mathrm{mdx}$ : $\mathrm{Cx} 43^{\mathrm{S3E} / \mathrm{S3E}}$ (mdxS3E) mice. The mice were backcrossed for at least 6 to 8 generations. Heterozygous intercrosses were maintained for all experiments. Genotyping for mdx and WT genotypes were screened for as described previously (92). Screening for $\mathrm{Cx} 43$ genotype was performed using custom-made forward primers (IDTDNA) that recognize either alanines, glutamic acids, or serines at the $325 / 328 / 330$ positions in $\mathrm{Cx} 43$ gene and a downstream common reverse sequence: $\mathrm{Cx} 43-\mathrm{S} 3 \mathrm{~A}$ : 5'-GCAACCATCGCCAACGCC-3'; Cx43-S3E: 5'-GAGAAACCATCGAGAACGAGCA-3'; Cx43-WT: 5'-AGGACCATCTCCAACTCC-3'; common reverse primer; 5'-CGCCTAGCTATCCCAAAACA-3'. Agematched WT and mdx control and $\mathrm{Cx} 43$-mutant male and female mice were analyzed at time points of 3 to 6 months (adult) and 14 to 18 months (aged). No significant differences were found between sexes in $\mathrm{Cx} 43$ remodeling or in response to Iso treatment (24).

Human samples. Three non-DMD and 3 DMD male human heart samples were obtained from the University of Maryland Brain and Tissue Bank, a member of the NIH NeuroBioBank network. All samples were dissected postmortem. DMD1's death was attributable to cardiac failure at age 15, DMD2's death was attributed to pulmonary thromboembolism at age 17, and DMD3's cause of death was unknown.

Western blotting. Snap-frozen mouse and human ventricular tissues were homogenized in RIPA buffer and processed as described (26). The following antibodies were used: $\mathrm{Cx} 43$ (MilliporeSigma, C6219; 1:10000, rabbit), pS325/S328/S330-Cx43 (custom made in-house, 1:1000, mouse), pS365-Cx43 (custom made in-house, 1:1000, rabbit), vinculin (MilliporeSigma, V9131; 1:2000, mouse), $N$-cadherin (Invitrogen, 33-3900; 1:2000, mouse), $\alpha$-tubulin (Abcam, ab7291; 1:2000, mouse), $\beta$-tubulin (Sigma T8328, 1:1000, mouse), gp91-phox (Santa Cruz Biotechnology Inc., sc-130543; 1:1000, mouse), CaMKIIס (GeneTex, GTX111401; 1:1000, rabbit), and oxidized-CaMKII (Met281/282, MilliporeSigma, 07-1387; 1:1000, rabbit). See Supplemental Methods for details.

Triton solubility assay. The method was adapted from a previous description (71) with modifications. See Supplemental Methods for details.

Ventricular cardiomyocyte isolation. Single ventricular cardiomyocytes were enzymatically isolated with collagenase type II (Worthington, LS004176) and protease XIV (MilliporeSigma, P5147) from 3- to 4-month-old mouse hearts using a Langendorff perfusion system previously described $(26,44)$.

Intracellular $\mathrm{Ca}^{2+}$ responses to hypo-osmotic shock. Intact cardiomyocytes were loaded with fluo-4AM ( $5 \mu \mathrm{M}$, Invitrogen), deesterified, and imaged as described previously (26). See Supplemental Methods for details.

Measurement of cytosolic ROS production. Intact cardiomyocytes were loaded with $5 \mu \mathrm{M} 2^{\prime}, 7^{\prime}$-dichlorofluorescen-diacetate ( $\mathrm{H}_{2}$-DCFA) and imaged for 120 seconds as described previously (26).

Tissue immunofluorescence. Mouse and human ventricular tissue were cut, processed, and incubated as described previously (24). Sec- 
tions were then incubated with either $\mathrm{Cx} 43$ (MilliporeSigma, C6219, 1:2000, rabbit) or $\mathrm{pS} 325 / \mathrm{S} 328 / \mathrm{S} 330-\mathrm{Cx} 43$ (see above, 1:1000) and $\mathrm{N}$-cadherin (Invitrogen 33-3900, 1:300, mouse) antibodies in blocking buffer overnight at $4^{\circ} \mathrm{C}$. See Supplemental Methods for more details.

Quantification of Cx43 localization at the intercalated disc. The method was adapted from a previous description (71) with modifications. See Supplemental Methods for more details.

Fibrosis staining and quantification. Masson Trichrome staining was performed as described previously (25).

Echocardiography. Transthoracic echocardiography of aged (14 to 18 months old) mice was performed and analyzed as described previously (25). Diaphragm ultrasonography was performed on anesthetized mice, and diaphragm amplitudes were measured as described previously (55).

Electrocardiography. Whole animal electrocardiograms were recorded in a lead II conformation and analyzed as described previously (24).

Isolated heart ethidium bromide perfusion and dye uptake quantification. Mice were heparinized (5000 U/ kg), anesthetized, and then injected with either saline (control) or Iso ( $5 \mathrm{mg} / \mathrm{kg}$, i.p.). Twenty minutes following Iso or vehicle injection, mice were sacrificed by cervical dislocation and hearts were extracted and cannulated in a Langendorff perfusion system. Hearts were perfused with $\mathrm{Ca}^{2+}$-free NT buffer at room temperature for 10 minutes, NT plus ethidium bromide $(5 \mu \mathrm{M})$ for 20 minutes, and then NT buffer for 5 minutes for wash out. Hearts were then fixed overnight in $4 \%$ paraformaldehyde (MilliporeSigma) and placed into 30\% sucrose solution in PBS (MilliporeSigma) for 6 hours, then embedded in OCT. After $10 \mu \mathrm{m}$ cryosections were made, slides were thawed to room temperature and washed in PBS; Alexa Fluor Wheat Germ Agglutinin 488 (Invitrogen) was applied for 20 minutes. Slides were then washed in PBS and mounted with Antifade Mounting Reagent with DAPI (Invitrogen). Slides were then imaged using a 200 Axiovert fluorescence microscope (Zeiss). Ethidium was red auto fluorescent, and thus the same exposure settings were used for all slides. To calculate ethidium fluorescence in Image (NIH), nuclei were identified and created as ROI. Individual nuclei (100 to 200 per image) mean fluorescent intensities were measured. Then the ROI outlines were projected onto corresponding ethidium images, where individual fluorescent intensities were measured, capturing the ethidium signal within all nuclei. Ethidium intensity was then divided by nuclei intensity per each ROI, after which the mean ratio was calculated for all nuclei in the image. From 2 to 3 images per heart were analyzed in a blinded fashion.

Isolated heart biotin perfusion. Mice were heparinized and anesthetized, then sacrificed by cervical dislocation. Hearts were extracted and cannulated in a Langendorff perfusion system. Hearts were initially perfused with NT for 5 minutes, switched to NT buffer plus Biotin (EZ-Link NHS Biotin, $0.5 \mathrm{mg} / \mathrm{mL}$, Thermo Scientific) for 40 minutes $(0.5 \mathrm{ml} / \mathrm{min}$ flow rate), and washed out for 10 minutes with NT buffer plus $15 \mathrm{mM}$ glycine. Ventricular tissue was then homogenized in HEN lysis buffer (250 mM HEPES, 1 mM EDTA, 0.1 mM Neucoproine, pH 8.0) with $2 \times$ HALT protease inhibitors (Thermo Scientific) and then centrifuged at $18,000 \mathrm{~g}$ for 5 minutes. Following protein concentration determination, $50 \mu \mathrm{l}$ of streptavidin beads (Thermo Scientific, 20349) were added to $200 \mu \mathrm{g}$ protein and nutated for 90 minutes at $4^{\circ} \mathrm{C}$ with occasional vortexing. Samples were then centrifuged at $18,000 \mathrm{~g}$ for 2 minutes and supernatant was discarded. The streptavidin pellet was then resuspended in fresh lysis buffer containing 0.1\% Triton X-100 and centrifuged for 1 minute at $18,000 \mathrm{~g}$. The pellet was then washed with PBS (pH 7.4) and centrifuged; $25 \mu$ of $2 \times$ Laemlli sample buffer was added and heated at $100^{\circ} \mathrm{C}$ for 5 minutes to disrupt biotin-streptavidin interaction. The heated samples were then centrifuged for $1 \mathrm{~min}-$ ute at $18,000 \mathrm{~g}$, and the supernatant was run along with total protein extracts without streptavidin pulldown on SDS-PAGE.

Mini osmotic pump implantation. Young adult (approximately 3 months old) mdx mice chosen for osmotic pump implantation were weighed and anesthetized. Osmotic pumps (Alzet model 1004) were inserted through a small incision between the scapulae, and the incision was then closed with wound clips. Osmotic pumps contained either saline (vehicle) or colch (MilliporeSigma, C9754, dissolved in saline, $0.4 \mathrm{mg} / \mathrm{kg} / \mathrm{d}$ ) and remained in the mice for 28 days. At the conclusion of the study, mice were euthanized, and cardiac tissue was collected for downstream immunoblotting and immunofluorescent analyses.

Statistics. Data are expressed as mean \pm SEM. Statistical analyses were performed using parametric analysis in GraphPad Prism software. Statistical significance between multiple groups was analyzed by 1-way ANOVA parametric testing followed by Tukey's multiple comparisons test. Statistical significance among mice that were injected with Iso or control was analyzed by 2-way ANOVA followed by Tukey's multiple comparisons test. In the case of 2 groups, we performed paired $t$ tests. $P$ values less than 0.05 were considered significant for all statistical tests. Representative $P$ values and symbols are described in the figure legends. Most experiments and analyses of endpoint readouts (immunohistochemistry, histopathology, immunoblotting) were carried out in a blinded fashion.

Study approval. All animal experiments were approved by the IACUC of Rutgers New Jersey Medical School and performed in accordance with NIH guidelines. All human experiments were approved by the IRB of Rutgers University and performed in accordance with relevant guidelines and regulations. Informed consent was obtained for all subjects from whom tissues were analyzed.

\section{Author contributions}

EH, MAL, DF, JEC, NS, and LHX designed experiments. EH and MAL performed most of the experiments. EH, MAL, DF, JEC, and NS analyzed the data. QZ, JPG, JN, HL, TL, and PDL performed some of the experiments. XHTW provided mdx:CaMKII (MM-VV) cardiac tissues, and PDL provided custom pS325/ S328/S330 antibody. GIF provided the transgenic S3A and S3E knockin mice. EH wrote the manuscript. All authors reviewed and approved the final draft.

\section{Acknowledgments}

We thank M. Zhu, L. Perez (Northeast Regional Alliance Program [NERA]), M.B. Ojeda (NERA), and P. Gupta for assistance in genotyping, histology and ECG reading; M. Teitelbaum for assistance in myocyte isolation; J. Jetko and P. Jetko for histology; and J. Sadoshima for critically reading the manuscript. This work was supported by an American Heart Association (AHA) predoctoral fellowship (17PRE33660354 to EH), an AHA postdoctoral fellowship (18POST339610107 to MAL), NIH grant 1R01HL141170-01 (to DF, NS, and JEC), NIH grant R01GM099490 (to JEC), NIH grant HL093342 (to NS), NIH grants R01HL92929 and R01Hl133294 (to LHX), NIH grant R01HL82727 (to GIF), AHA grant 16GRNT31100022 (to LHX), NIH grant GM55632 (to PDL), NIH grants R01GM112415 and P30NS046593 (to HL), and Muscular 
Dystrophy Association grants 602349 and 416281 (to DF). The mass spectrometry data were obtained from an Orbitrap instrument funded in part by NIH grant NSO46593 for the support of the Rutgers Mass Spectrometry Center for Integrative Neuroscience Research.
Address correspondence to: Diego Fraidenraich, Medical Sciences Building G-667, 185 South Orange Avenue, Newark, New Jersey 07103, USA. Phone: 973.972.5525; Email: fraidedi@ njms.rutgers.edu.
1. Bushby K, et al. Diagnosis and management of Duchenne muscular dystrophy, part 1: diagnosis, and pharmacological and psychosocial management. Lancet Neurol. 2010;9(1):77-93.

2. Kamdar F, Garry DJ. Dystrophin-deficient cardiomyopathy. JAm Coll Cardiol. 2016;67(21):2533-2546.

3. Hoffman EP, Brown RH, Kunkel LM. Dystrophin: the protein product of the Duchenne muscular dystrophy locus. Cell. 1987;51(6):919-928.

4. Lapidos KA, Kakkar R, McNally EM. The dystrophin glycoprotein complex: signaling strength and integrity for the sarcolemma. Circ Res. 2004;94(8):1023-1031.

5. Le Guiner C, et al. Long-term microdystrophin gene therapy is effective in a canine model of Duchenne muscular dystrophy. Nat Commun. 2017;8:16105.

6. Long C, et al. Correction of diverse muscular dystrophy mutations in human engineered heart muscle by single-site genome editing. Sci $A d v$. 2018;4(1):eaap9004.

7. Kieny P, et al. Evolution of life expectancy of patients with Duchenne muscular dystrophy at AFM Yolaine de Kepper centre between 1981 and 2011. Ann Phys Rehabil Med. 2013;56(6):443-454.

8. McNally EM. New approaches in the therapy of cardiomyopathy in muscular dystrophy. Annu Rev Med. 2007;58:75-88.

9. Ogata H, Ishikawa Y, Ishikawa Y, Minami R. Beneficial effects of beta-blockers and angiotensin-converting enzyme inhibitors in Duchenne muscular dystrophy. J Cardiol. 2009;53(1):72-78.

10. Laird DW, Lampe PD. Therapeutic strategies targeting connexins. Nat Rev Drug Discov. 2018;17(12):905-921.

11. Kleber AG, Saffitz JE. Role of the intercalated disc in cardiac propagation and arrhythmogenesis. Front Physiol. 2014;5:404.

12. Michela P, Velia V, Aldo P, Ada P. Role of connexin 43 in cardiovascular diseases. Eur JPharmacol. 2015;768:71-76.

13. Lampe PD, Lau AF. The effects of connexin phosphorylation on gap junctional communication. Int J Biochem Cell Biol. 2004;36(7):1171-1186.

14. Aasen T, Johnstone S, Vidal-Brime L, Lynn KS, Koval M. Connexins: synthesis, post-translational modifications, and trafficking in health and disease. Int J Mol Sci. 2018;19(5):E1296.

15. Smith JH, Green CR, Peters NS, Rothery S, Severs NJ. Altered patterns of gap junction distribution in ischemic heart disease. An immunohistochemical study of human myocardium using laser scanning confocal microscopy. Am J Pathol. 1991;139(4):801-821.

16. Wang N, et al. Selective inhibition of $\mathrm{Cx} 43$ hemichannels by Gap19 and its impact on myocardial ischemia/reperfusion injury. Basic Res Cardiol. 2013;108(1):309.

17. Hawat G, Hélie P, Baroudi G. Single intravenous low-dose injections of connexin 43 mimetic peptides protect ischemic heart in vivo against myocardial infarction. J Mol Cell Cardiol. 2012;53(4):559-566.

18. $\mathrm{Qu} \mathrm{J}$, et al. Gap junction remodeling and spironolactone-dependent reverse remodeling in the hypertrophied heart. Circ Res. 2009;104(3):365-371.

19. Huang RY, et al. Identification of CaMKII phosphorylation sites in Connexin 43 by high-resolution mass spectrometry. J Proteome Res. 2011;10(3):1098-1109.

20. Remo BF, et al. Phosphatase-resistant gap junctions inhibit pathological remodeling and prevent arrhythmias. Circ Res. 2011;108(12):1459-1466.

21. Severs NJ, Bruce AF, Dupont E, Rothery S. Remodelling of gap junctions and connexin expression in diseased myocardium. Cardiovasc Res. 2008;80(1):9-19.

22. Fontes MS, van Veen TA, de Bakker JM, van Rijen HV. Functional consequences of abnormal Cx43 expression in the heart. Biochim Biophys Acta. 2012;1818(8):2020-2029.

23. Boulaksil M, et al. Spatial heterogeneity of $\mathrm{Cx} 43$ is an arrhythmogenic substrate of polymorphic ventricular tachycardias during compensated cardiac hypertrophy in rats. Front Cardiovasc Med. 2016;3:5.

24. Gonzalez JP, Ramachandran J, Xie LH, Contreras JE, Fraidenraich D. Selective connexin 43 inhibition prevents isoproterenol-induced arrhythmias and lethality in muscular dystrophy mice. Sci Rep. 2015;5:13490.

25. Gonzalez JP, et al. Small fractions of muscular dystrophy embryonic stem cells yield severe cardiac and skeletal muscle defects in adult mouse chimeras. Stem Cells. 2017;35(3):597-610.

26. Gonzalez JP, et al. Normalization of connexin 43 protein levels prevents cellular and functional signs of dystrophic cardiomyopathy in mice. $\mathrm{Neu}$ romuscul Disord. 2018;28(4):361-372.

27. Solan JL, Lampe PD. Key connexin 43 phosphorylation events regulate the gap junction life cycle. JMembr Biol. 2007;217(1-3):35-41.

28. Shirokova N, Niggli E. Cardiac phenotype of Duchenne Muscular Dystrophy: insights from cellular studies. J Mol Cell Cardiol. 2013;58:217-224.

29. Erickson JR, He BJ, Grumbach IM, Anderson ME. CaMKII in the cardiovascular system: sensing redox states. Physiol Rev. 2011;91(3):889-915.

30. Lampe PD, Cooper CD, King TJ, Burt JM. Analysis of Connexin 43 phosphorylated at S325, S328 and $\mathrm{S} 330$ in normoxic and ischemic heart. J Cell Sci. 2006;119(Pt 16):3435-3442.

31. Colussi $\mathrm{C}$, et al. $\mathrm{N} \varepsilon$-lysine acetylation determines dissociation from GAP junctions and lateralization of connexin 43 in normal and dystrophic heart. Proc Natl Acad Sci USA. 2011;108(7):2795-2800.

32. Cooper CD, Lampe PD. Casein kinase 1 regulates connexin-43 gap junction assembly. J Biol Chem. 2002;277(47):44962-44968.

33. Musil LS, Goodenough DA. Biochemical analysis of connexin 43 intracellular transport, phosphorylation, and assembly into gap junctional plaques. J Cell Biol. 1991;115(5):1357-1374.

34. Chenard AA, Becane HM, Tertrain F, de Kermadec JM, Weiss YA. Ventricular arrhythmia in Duchenne muscular dystrophy: prevalence, significance and prognosis. Neuromuscul Disord. 1993;3(3):201-206.

35. Kerr JP, et al. Detyrosinated microtubules modulate mechanotransduction in heart and skeletal muscle. Nat Commun. 2015;6:8526.

36. Chu V, et al. Electrocardiographic findings in $\mathrm{mdx}$ mice: a cardiac phenotype of Duchenne muscular dystrophy. Muscle Nerve. 2002;26(4):513-519.

37. Fauconnier J, et al. Leaky RyR2 trigger ventricular arrhythmias in Duchenne muscular dystrophy. Proc Natl Acad Sci USA. 2010;107(4):1559-1564.

38. Gavillet B, et al. Cardiac sodium channel Nav1.5 is regulated by a multiprotein complex composed of syntrophins and dystrophin. Circ Res. 2006;99(4):407-414.

39. Petitprez S, et al. SAP97 and dystrophin macromolecular complexes determine two pools of cardiac sodium channels Nav1.5 in cardiomyocytes. Circ Res. 2011;108(3):294-304.

40. Contreras JE, Sáez JC, Bukauskas FF, Bennett MV. Gating and regulation of connexin 43 (Cx43) hemichannels. Proc Natl Acad Sci USA. 2003;100(20):11388-11393.

41. Sáez JC, Schalper KA, Retamal MA, Orellana JA, Shoji KF, Bennett MV. Cell membrane permeabilization via connexin hemichannels in living and dying cells. Exp Cell Res. 2010;316(15):2377-2389.

42. De Vuyst E, et al. $\mathrm{Ca}(2+)$ regulation of connexin 43 hemichannels in C6 glioma and glial cells. Cell Calcium. 2009;46(3):176-187.

43. Braet K, Vandamme W, Martin PE, Evans WH, Leybaert L. Photoliberating inositol-1,4,5-trisphosphate triggers ATP release that is blocked by the connexin mimetic peptide gap 26. Cell Calcium. 2003;33(1):37-48.

44. Fanchaouy M, Polakova E, Jung C, Ogrodnik J, Shirokova N, Niggli E. Pathways of abnormal stress-induced $\mathrm{Ca} 2+$ influx into dystrophic $\mathrm{mdx}$ cardiomyocytes. Cell Calcium. 2009;46(2):114-121.

45. Ullrich ND, Fanchaouy M, Gusev K, Shirokova N, Niggli E. Hypersensitivity of excitation-contraction coupling in dystrophic cardiomyocytes. Am JPhysiol Heart Circ Physiol. 2009;297(6):H1992-H2003.

46. Williams IA, Allen DG. The role of reactive oxygen species in the hearts of dystrophin-deficient mdx mice. Am J Physiol Heart Circ Physiol. 2007;293(3):H1969-H1977.

47. Ramachandran S, Xie LH, John SA, Subramaniam S, Lal R. A novel role for connexin hemichannel in oxidative stress and smoking-induced cell injury. PLoS One. 2007;2(8):e712.

48. Retamal MA, Schalper KA, Shoji KF, Bennett MV, Sáez JC. Opening of connexin 43 hemichannels is increased by lowering intracellular redox potential. Proc Natl Acad Sci USA. 2007;104(20):8322-8327.

49. Le HT, et al. Gap junction intercellular communication mediated by connexin 43 in astrocytes is 
essential for their resistance to oxidative stress. J Biol Chem. 2014;289(3):1345-1354.

50. Bedard K, Krause KH. The NOX family of ROS-generating NADPH oxidases: physiology and pathophysiology. Physiol Rev. 2007;87(1):245-313.

51. Ather S, Wang W, Wang Q, Li N, Anderson ME, Wehrens XH. Inhibition of CaMKII phosphorylation of RyR2 prevents inducible ventricular arrhythmias in mice with Duchenne muscular dystrophy. Heart Rhythm. 2013;10(4):592-599.

52. Wang $\mathrm{Q}$, Wang W, Wang G, Rodney GG, Wehrens XH. Crosstalk between RyR2 oxidation and phosphorylation contributes to cardiac dysfunction in mice with Duchenne muscular dystrophy. JMol Cell Cardiol. 2015;89(Pt B):177-184.

53. Wang Q, et al. Oxidized CaMKII $\left(\mathrm{Ca}^{2+} /\right.$ calmodulin-dependent protein kinase ii) is essential for ventricular arrhythmia in a mouse model of Duchenne muscular dystrophy. Circ Arrhythm Electrophysiol. 2018;11(4):e005682.

54. Quinlan JG, Hahn HS, Wong BL, Lorenz JN, Wenisch AS, Levin LS. Evolution of the $\mathrm{mdx}$ mouse cardiomyopathy: physiological and morphological findings. Neuromuscul Disord. 2004;14(8-9):491-496.

55. Whitehead NP, Bible KL, Kim MJ, Odom GL, Adams ME, Froehner SC. Validation of ultrasonography for non-invasive assessment of diaphragm function in muscular dystrophy. J Physiol (Lond). 2016;594(24):7215-7227.

56. Stedman HH, et al. The mdx mouse diaphragm reproduces the degenerative changes of Duchenne muscular dystrophy. Nature. 1991;352(6335):536-539.

57. Lefaucheur JP, Pastoret C, Sebille A. Phenotype of dystrophinopathy in old $\mathrm{mdx}$ mice. Anat Rec. 1995;242(1):70-76.

58. Belanto JJ, et al. Microtubule binding distinguishes dystrophin from utrophin. Proc Natl Acad Sci USA. 2014;111(15):5723-5728.

59. Prosser BL, Ward CW, Lederer WJ. X-ROS signaling: rapid mechano-chemo transduction in heart. Science. 2011;333(6048):1440-1445.

60. Prosser BL, Khairallah RJ, Ziman AP, Ward CW, Lederer WJ. X-ROS signaling in the heart and skeletal muscle: stretch-dependent local ROS regulates [Ca ${ }^{2+}$ ]. J Mol Cell Cardiol. 2013;58:172-181.

61. Joca HC, Coleman AK, Ward CW, Williams GSB. Quantitative tests reveal that microtubules tune the healthy heart but underlie arrhythmias in pathology [published online ahead of print December 24, 2018]. J Physiol. https://doi. org/10.1113/JP277083.

62. Loehr JA, et al. NADPH oxidase mediates microtubule alterations and diaphragm dysfunction in dystrophic mice. Elife. 2018;7:e31732.

63. Giepmans BN, et al. Gap junction protein connexin-43 interacts directly with microtubules. Curr Biol. 2001;11(17):1364-1368.
64. Smyth JW, et al. Limited forward trafficking of connexin 43 reduces cell-cell coupling in stressed human and mouse myocardium. J Clin Invest. 2010;120(1):266-279.

65. Chkourko HS, et al. Remodeling of mechanical junctions and of microtubule-associated proteins accompany cardiac connexin 43 lateralization. Heart Rhythm. 2012;9(7):1133-1140.e6.

66. Prins KW, Asp ML, Zhang H, Wang W, Metzger JM. Microtubule-mediated misregulation of junctophilin-2 underlies T-tubule disruptions and calcium mishandling in $m d x$ mice. JACC Basic Transl Sci. 2016;1(3):122-130.

67. Khairallah RJ, et al. Microtubules underlie dysfunction in duchenne muscular dystrophy. Sci Signal. 2012;5(236):ra56.

68. Sakurai T, Tsuchida M, Lampe PD, Murakami M. Cardiomyocyte FGF signaling is required for $\mathrm{Cx} 43$ phosphorylation and cardiac gap junction maintenance. Exp Cell Res. 2013;319(14):2152-2165.

69. Wang WE, et al. Dedifferentiation, proliferation, and redifferentiation of adult mammalian cardiomyocytes after ischemic injury. Circulation. 2017;136(9):834-848.

70. Smyth JW, et al. Actin cytoskeleton rest stops regulate anterograde traffic of connexin 43 vesicles to the plasma membrane. Circ Res. 2012;110(7):978-989.

71. Basheer WA, et al. GJA1-20k arranges actin to guide $\mathrm{Cx} 43$ delivery to cardiac intercalated discs. Circ Res. 2017;121(9):1069-1080.

72. Campbell KP. Three muscular dystrophies: loss of cytoskeleton-extracellular matrix linkage. Cell. 1995;80(5):675-679.

73. Rybakova IN, Amann KJ, Ervasti JM. A new model for the interaction of dystrophin with F-actin. JCell Biol. 1996;135(3):661-672.

74. Saidi Brikci-Nigassa A, et al. Phosphorylation controls the interaction of the connexin $43 \mathrm{C}$-terminal domain with tubulin and microtubules. Biochemistry. 2012;51(21):4331-4342.

75. Lin R, Warn-Cramer BJ, Kurata WE, Lau AF. v-Src phosphorylation of connexin 43 on Tyr247 and Tyr265 disrupts gap junctional communication. JCell Biol. 2001;154(4):815-827.

76. Gervásio OL, Whitehead NP, Yeung EW, Phillips WD, Allen DG. TRPC1 binds to caveolin-3 and is regulated by Src kinase - role in Duchenne muscular dystrophy. JCell Sci. 2008;121(Pt 13):2246-2255

77. Bačová $\mathrm{B}$, et al. Up-regulation of myocardial connexin- 43 in spontaneously hypertensive rats fed red palm oil is most likely implicated in its anti-arrhythmic effects. Can J Physiol Pharmacol. 2012;90(9):1235-1245.

78. Dhein S, et al. Improving cardiac gap junction communication as a new antiarrhythmic mechanism: the action of antiarrhythmic peptides. Naunyn Schmiedebergs Arch Pharmacol. 2010;381(3):221-234.

79. Sovari AA, et al. Inhibition of c-Src tyrosine kinase prevents angiotensin II-mediated connexin- 43 remodeling and sudden cardiac death. J Am Coll Cardiol. 2011;58(22):2332-2339.

80. Melacini P, et al. Cardiac and respiratory involvement in advanced stage Duchenne muscular dystrophy. Neuromuscul Disord. 1996;6(5):367-376.

81. Merrifield PA, Laird DW. Connexins in skeletal muscle development and disease. Semin Cell Dev Biol. 2016;50:67-73.

82. Cea LA, et al. Fast skeletal myofibers of $\mathrm{mdx}$ mouse, model of Duchenne muscular dystrophy, express connexin hemichannels that lead to apoptosis. Cell Mol Life Sci. 2016;73(13):2583-2599.

83. Cea LA, et al. De novo expression of connexin hemichannels in denervated fast skeletal muscles leads to atrophy. Proc Natl Acad Sci USA. 2013;110(40):16229-16234.

84. Guido AN, Campos GE, Neto HS, Marques MJ, Minatel E. Fiber type composition of the sternomastoid and diaphragm muscles of dystrophin-deficient mdx mice. Anat Rec (Hoboken). 2010;293(10):1722-1728.

85. Ek-Vitorín JF, Pontifex TK, Burt JM. Cx43 channel gating and permeation: multiple phosphorylation-dependent roles of the carboxyl terminus. Int J Mol Sci. 2018;19(6):E1659.

86. Macquart C, et al. Microtubule cytoskeleton regulates connexin 43 localization and cardiac conduction in cardiomyopathy caused by mutation in A-type lamins gene [published online ahead of print June 8, 2018]. Hum Mol Genet. https://doi. org/10.1093/hmg/ddy227.

87. Chen CY, et al. Suppression of detyrosinated microtubules improves cardiomyocyte function in human heart failure. Nat Med. 2018;24(8):1225-1233.

88. Gwizdala A, et al. Safety, feasibility and effectiveness of first in-human administration of muscle-derived stem/progenitor cells modified with connexin- 43 gene for treatment of advanced chronic heart failure. Eur J Heart Fail. 2017;19(1):148-157.

89. Raman SV, et al. Eplerenone for early cardiomyopathy in Duchenne muscular dystrophy: results of a two-year open-label extension trial. Orphanet J Rare Dis. 2017;12(1):39.

90. Kobayashi N, et al. Cardioprotective mechanisms of eplerenone on cardiac performance and remodeling in failing rat hearts. Hypertension. 2006;47(4):671-679.

91. Crassous PA, et al. Newly identified NO-sensor guanylyl cyclase/connexin 43 association is involved in cardiac electrical function. J Am Heart Assoc. 2017;6(12):e006397.

92. Grange RW, Gainer TG, Marschner KM, Talmadge RJ, Stull JT. Fast-twitch skeletal muscles of dystrophic mouse pups are resistant to injury from acute mechanical stress. Am J Physiol, Cell Physiol. 2002;283(4):C1090-C1101. 